\title{
Estrogen-mediated gut microbiome alterations influence sexual dimorphism in metabolic syndrome in mice
}

\author{
Kanakaraju Kaliannan ${ }^{1}$, Ruairi C. Robertson ${ }^{2,3,4}$, Kiera Murphy² ${ }^{2}$ Catherine Stanton ${ }^{2,3,4}$, Chao Kang ${ }^{5}$, Bin Wang ${ }^{5}$, \\ Lei Hao ${ }^{1}$, Atul K. Bhan ${ }^{6}$ and Jing X. Kang ${ }^{1 *}$
}

\begin{abstract}
Background: Understanding the mechanism of the sexual dimorphism in susceptibility to obesity and metabolic syndrome (MS) is important for the development of effective interventions for MS.

Results: Here we show that gut microbiome mediates the preventive effect of estrogen (17 $\beta$-estradiol) on metabolic endotoxemia (ME) and low-grade chronic inflammation (LGCl), the underlying causes of MS and chronic diseases. The characteristic profiles of gut microbiome observed in female and $17 \beta$-estradiol-treated male and ovariectomized mice, such as decreased Proteobacteria and lipopolysaccharide biosynthesis, were associated with a lower susceptibility to ME, LGCl, and MS in these animals. Interestingly, fecal microbiota-transplant from male mice transferred the MS phenotype to female mice, while antibiotic treatment eliminated the sexual dimorphism in MS, suggesting a causative role of the gut microbiome in this condition. Moreover, estrogenic compounds such as isoflavones exerted microbiomemodulating effects similar to those of $17 \beta$-estradiol and reversed symptoms of MS in the male mice. Finally, both expression and activity of intestinal alkaline phosphatase (IAP), a gut microbiota-modifying non-classical anti-microbial peptide, were upregulated by $17 \beta$-estradiol and isoflavones, whereas inhibition of IAP induced ME and LGCI in female mice, indicating a critical role of IAP in mediating the effects of estrogen on these parameters.

Conclusions: In summary, we have identified a previously uncharacterized microbiome-based mechanism that sheds light upon sexual dimorphism in the incidence of MS and that suggests novel therapeutic targets and strategies for the management of obesity and MS in males and postmenopausal women.
\end{abstract}

Keywords: Estrogen, Gut microbiome, Obesity, Metabolic syndrome, Isoflavones, Chronic inflammation

\section{Background}

Metabolic syndrome (MS) is a cluster of metabolic abnormalities including obesity, visceral adiposity, hyperinsulinemia, hyperglycemia, hypertension, and hypercholesterolemia [1]. It is a leading health issue facing western societies owing to the high sucrose, high saturated fat content, and elevated omega-6/omega-3 fatty acid ratio of the western diet (WD) [2]. Sexual dimorphism in obesity and metabolic dysfunction are observed in both experimental animal models of MS [3-5] and in humans [6]. In fact, in many rodent models, insulin resistance occurs rarely in females or

\footnotetext{
* Correspondence: kang.jing@mgh.harvard.edu; jxkang@mgh.harvard.edu 'Laboratory of Lipid Medicine and Technology, Department of Medicine, Massachusetts General Hospital and Harvard Medical School, 149 -13th Street, Boston, MA 02129, USA

Full list of author information is available at the end of the article
}

exclusively in males [3]. Moreover, protection from severe high-fat diet (HFD)-induced obesity and MS in C57BL/6 female mice precludes the interrogation of disease pathogenesis in a sex-independent manner [7]. Sex steroid hormones are believed to underlie sexual dimorphism in metabolic outcomes in response to stressors such as the WD, with estrogens theorized to protect women until menopause [8]. Supporting this position, the prevalence of MS is higher in men than in similarly aged pre-menopausal women [6] and a higher level of adiposity is required in women to elicit metabolic disturbances [9]. Conversely, following the menopause, women tend to accumulate visceral fat and become more insulin resistant, with a consequent increase in the risk of type 2 diabetes [10]. An 
increasing body of evidence suggests that estrogens also have important beneficial effects on body fat and metabolism in males $[10,11]$.

The gut microbiota comprises trillions of bacteria that contribute to nutrient acquisition and energy regulation $[12,13]$. Growing evidence indicates that obesity is closely linked with low-grade chronic inflammation (LGCI), which can lead to MS [14, 15]. In addition, changes in the composition of the gut microbiota are known to be associated with the development of obesity and its associated metabolic disorders [16]. Interestingly, an increased ratio of the major phyla Firmicutes and Bacteroidetes (FIR/BAC ratio) and depletion of several bacterial species (e.g., Akkermansia mucinophilia) can promote the development of obesity in both dietary and genetic models of obesity in mice [17-19]. Other studies in animal models of obesity suggest that obesity-induced gut dysbiosis caused by either environmental or genetic factors increase populations of bacteria that produce the endotoxin lipopolysaccharide (LPS) [14] and decrease LPS-suppressing bacteria [20, 21]. This process leads to impaired gut barrier integrity and release of LPS from intestinal gram-negative bacteria into the bloodstream $[14,22]$ which in turn leads to Toll-like receptor 4 (TLR4)-mediated metabolic endotoxemia (ME), LGCI and insulin resistance in obese mice [23, 24]. Moreover, chronic injection of LPS in mice causes mild obesity and insulin resistance [23], highlighting a possible role for microbiota-derived LPS in obesity-induced inflammation.

The causative role of the gut microbiota in the context of MS is well characterized [14, 20,21], but the role of sexual dimorphism on the composition of gut microbiota in the context of MS, and the associated mechanisms underlying such differences, are still unclear. Here, we report that sexual dimorphism in MS is associated with estrogen-mediated changes in the gut microbiome, ME and LGCI, and that $17 \beta$-estradiol $(17 \beta-E)$ (E2) treatment prevents MS in male and ovariectomized (OVX) mice by altering gut microbiome and intestinal alkaline phosphatase (IAP), a major gut microbiota-modifying enzyme. Our results shed light on distinct male and female profiles for gut microbiome, IAP, and markers of ME and LGCI that may contribute to sexual dimorphism in MS, revealing new possibilities for preventing and controlling human obesity-related metabolic dysfunction in males and postmenopausal women.

\section{Results}

Sex differences in ME and LGCI are associated with sexual dimorphism in MS

We found that relative to males $(n=11)$, female mice $(n=$ 11) had significantly lower levels of markers of WD-induced obesity, including gross appearance of mice, intra-abdominal and gonadal fat distribution, body weight gain, and white adipose tissues accumulation) (Additional file 1: Figure S1a-c). Noticeably, although there were no differences in energy intake between males and females (Additional file 1: Figure S1d), relative to males, females had improved MS parameters such as glucose intolerance (glucose tolerance test with area under the curve and HOMA-IR) (Additional file 1: Figure S1e), non-alcoholic fatty liver disease (Additional file 1: Figure S1F-I), and dyslipidemia (total serum cholesterol, triglyceride, HDL-C, LDL-C, and atherogenic index) (Additional file 1: Figure S1j-n). We then investigated whether oral 17ß-E (E2) administration ameliorated WD-induced MS in male and ovariectomized (OVX) mice $(n=5$ per group). Interestingly, MS parameters were more evident in OVX mice compared to normal female (F) mice, and the administration of $17 \beta-E$ significantly suppressed the development of MS in the male (male+E2) and OVX (OVX+E2) groups (Fig 1a-o and Additional file 1: Figure S1y-z), irrespective of energy intake (Additional file 1: Figure S1x). Notably, there were no significant differences in MS parameters between the female and male+E2 groups (Fig. 1a-o and Additional file 1: Figure S1y-z). This was accompanied with elevated levels of serum $17 \beta-E$ concentrations in male $+\mathrm{E} 2$ and $\mathrm{OVX}+\mathrm{E} 2$ groups treated with $17 \beta-\mathrm{E}$ compared to control male and OVX groups respectively (Additional file 1: Figure S1p). We investigated whether sexual dimorphism in MS is associated with sex-specific differences in the markers of ME [serum LPS, LPS-binding protein (LBP), soluble CD14 (sCD14), and intestinal permeability] and LGCI [tumor necrosis factor- $\alpha$ (TNF- $\alpha$ ), interleukin-1 $\beta$ (IL-1 $\beta$ ), IL-6, monocyte chemoattractant protein-1 (MCP-1) and IL-10], and whether estrogen plays a key role in this context. Surprisingly, we found significant differences in the markers of ME (Fig. 1q-t and Additional file 1: Figure S1o-r) and LGCI (Fig. 1u-y and Additional file 1: Figure S1s-w) between WD-fed male and female mice. Lack of endogenous estrogen support in the OVX mice induced significantly elevated levels of markers of ME (Fig. 1q-t) and LGCI (Fig. $1 \mathrm{u}-\mathrm{y}$ ) compared to the female group. Moreover, 17 $\beta-\mathrm{E}$ treatment reduced the occurrence of ME and LGCI in the $\mathrm{M}+\mathrm{E} 2$ and $\mathrm{OVX}+\mathrm{E} 2$ groups to levels equal to those female mice (Fig. 1q-r).

\section{Gut microbiome mediates the development of metabolic syndrome in a sex-specific manner}

Given that metabolic endotoxemia is commonly derived from gut dysbiosis, we next used high-throughput $16 \mathrm{~S}$ rRNA gene sequencing to determine whether sexual dimorphism exist in gut microbiome, and whether $17 \beta-E$ treatment affected the microbiome composition. V3-V4 $16 \mathrm{~S}$ rRNA gene sequencing was performed on fecal samples collected from $\mathrm{M}, \mathrm{F}, \mathrm{M}+\mathrm{E} 2$, OVX, and OVX+E2 groups ( $n=5$ per group) that were fed a WD. Principal coordinate (PCoA) analysis of a common $\beta$-diversity 
a

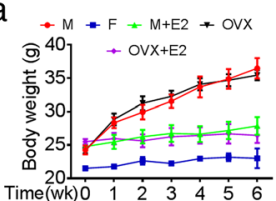

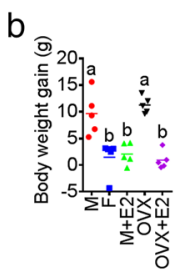

f

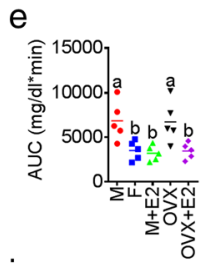

i

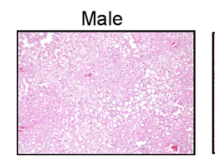

k
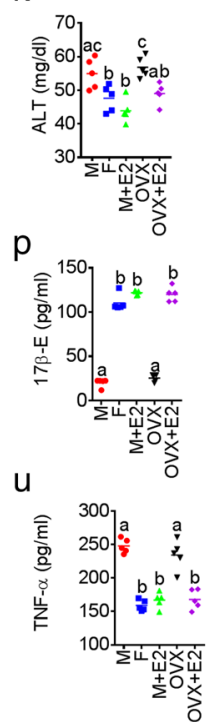

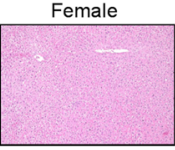

I

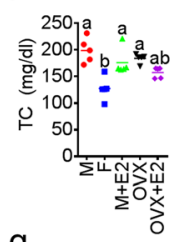

q
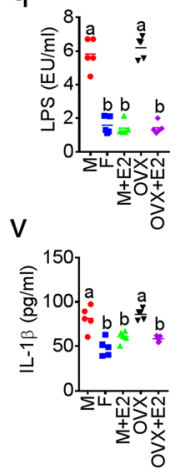
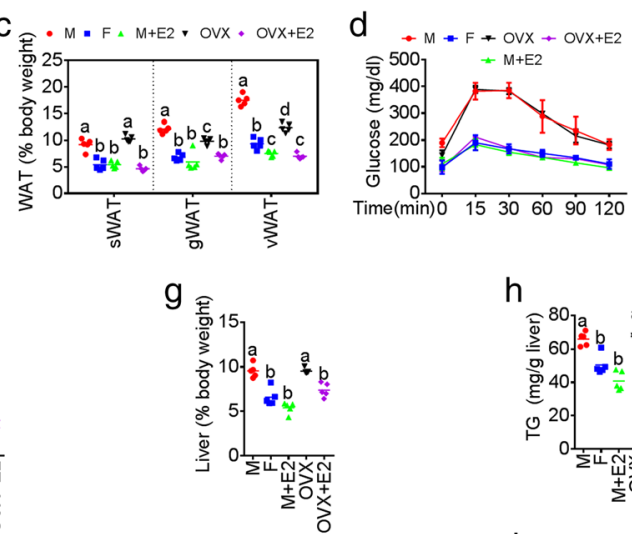

h
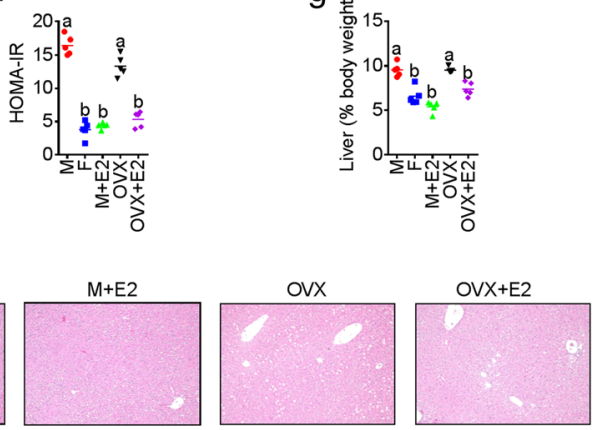

$\mathrm{m}$

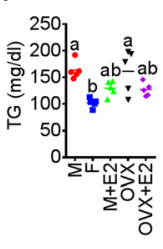

$r$

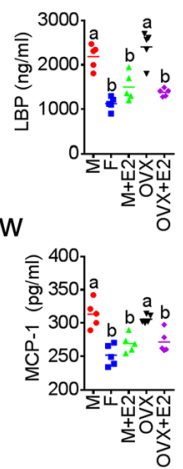

$\mathrm{n}$
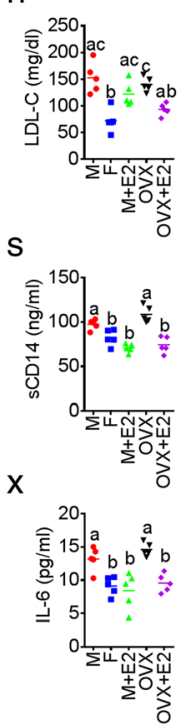

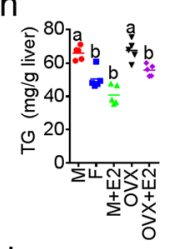

j

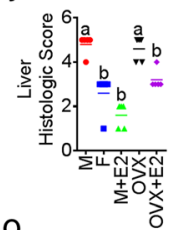

0
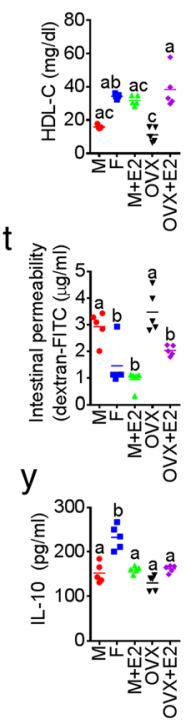

Fig. 1 Sexual differences in ME and LGCl are associated with sexual dimorphism in obesity and MS. Male (M), normal female (F), and ovariectomized $(\mathrm{OVX})$ female mice were divided in to five groups (M, F, M+E2, OVX, and OVX+E2; $n=5 /$ group) and were fed western diet (WD) $\pm 17 \beta$-estradiol (E2) in the drinking water for 6 weeks. Markers for sexual dimorphism in metabolic syndrome (MS) (a-o), metabolic endotoxemia (ME) (q-t), and low-grade chronic inflammation (LGCl) (u-y) were studied. a Weekly body weight. b Body weight gain. $\mathbf{c}$ White adipose tissue (WAT) weight. $\mathbf{d}$ Glucose tolerance test (GTT). e GTT area under curve (AUC). $\mathbf{f}$ Homeostatic model assessment-insulin resistance (HOMA-IR). $\mathbf{g}$ Liver weight. $\mathbf{h}$ Liver triglyceride. $\mathbf{i}$ Hematoxylin and eosin-stained liver specimens showing histological evidences for fatty liver. $\mathbf{j}$ Fatty liver score. Serum levels of alanine aminotransferase (ALT) (k), total cholesterol (TC) (I), triglyceride (TG) (m), low-density lipoprotein cholesterol (LDL-C) (n), high-density lipoprotein cholesterol (HDL-C) (o), E2 levels (p), lipopolysaccharides (LPS) (q), LPS-binding proteins (LBP) (r), and soluble CD14 (sCD14) (s). t Serum levels of FITC-dextran macromolecules indicating

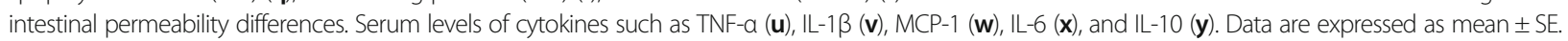
Box plots (box showing the median, and the 25th and 75th percentiles, and the whiskers of the graph show the largest and smallest values) were used. Data with different superscript letters are significantly different $(P<0.05)$. Ordinary or repeated measures one-way ANOVA followed by Tukey's multiple comparisons test

index (Bray-Curtis distance) applied on whole microbiota abundance identified a distinct clustering of microbiota composition between male and OVX female and F/OVX $+\mathrm{E} 2 / \mathrm{M}+\mathrm{E} 2$ groups along the primary ordination axis (axis 1), results are shown in Fig. 2a and Additional file 1: Table
S1. Next, taxa which are primarily responsible for an observed difference between groups were analyzed using the SIMPER test (similarity percentage analysis), which identified 10 taxa that were annotated at the genus level. PCoA showed that gram-negative LPS-producing Escherichial 

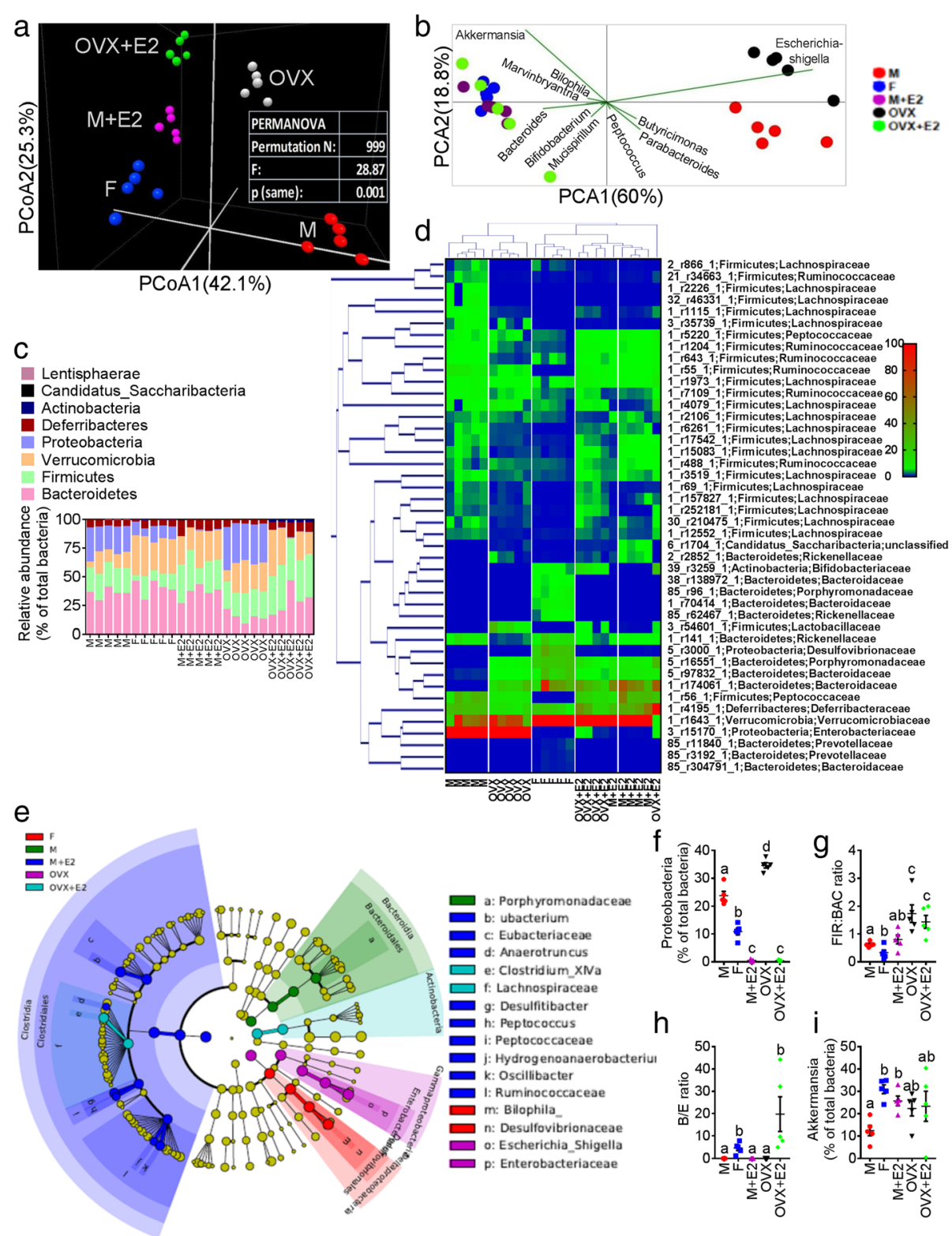

Fig. 2 Estrogen alters the gut microbiome. Male (M), normal female (F), and ovariectomized (OVX) female mice were divided in to five groups (M, $F, M+E 2, O V X$, and $O V X+E 2 ; n=5 / g r o u p)$ and were fed western diet (WD) $\pm 17 \beta$-estradiol (E2) in the drinking water for 6 weeks. a $\beta$-diversity analysis of whole microbiota relative abundance (RA) using principal coordinate analysis (PCOA) with Bray-Curtis dissimilarity index (BCD) followed by permutational multivariate analysis of variance (PERMANOVA) significance test. $\mathbf{b}$ Similarity percentage (SIMPER) analysis with BCD was used to identify the specific genera with the greatest contribution to the differences observed between the groups, followed by principal component analysis (PCA) (variance-covariance type) showing the top 10 operational taxonomic unit (OTU) scores included as vectors. The magnitude and direction correspond to the weights. c Analysis at the phylum level using RA (\%). $\mathbf{d}$ Hierarchical clustering with a heat map shows the RA of representative OTUs (those with greatest difference between five groups) group means from each family selected for $P<0.05$, obtained with differential abundance analysis. The OTUs are shown as OTU number, phylum and family. e Cladogram generated from linear discriminant analysis (LDA) effect size (LEfSe) showing the relationship between taxon (the levels represent, from the inner to outer rings, phylum, class, order, family, and genus). $\mathbf{f}-\mathbf{i}$ Scatter plots showing the phylum Proteobacteria, Firmicutes (FIR) to Bacteroidetes (BAC) ratio, Bifidobacterium (B) to Enterobacteriacea (E) ratio and RA of genus Akkermansia (\%). Data was shown as mean \pm SEM. Data with different superscript letters are significantly different $(P<0.05)$. One-way ANOVA followed by Tukey's multiple comparisons test

Shigella largely contributed to M/OVX groups, whereas LPS-suppressing Akkermansia muciniphila [14, 25] and Bifidobacterium $[14,26]$ were associated with the female and
17ß-E-treated M/OVX groups (Fig. 2b). At the phylum level, the majority of the bacterial phyla identified in the fecal samples were encompassed by Bacteriodetes, Firmicutes, 
Proteobacteria, and Verrucomicrobia, as shown in Fig. 2c. Furthermore, the relative abundance (RA) of taxa, which showed false discovery rate (FDR)-corrected $P$ value $<0.05$ with differential expression analysis conducted on whole microbiota profile, were expressed as heat map (Fig. 2d and Additional file 1: Table S2), including hierarchical clustering $(\mathrm{HCN}) . \mathrm{HCN}$ is a clustering technique for graphically summarizing the inter-sample relationships in the form of a dendrogram. $\mathrm{HCN}$ also clearly separated the male/OVX samples as a single cluster from the other three groups (F, $\mathrm{M}+\mathrm{E} 2$, OVX+E2) (Additional file 1: Figure S2a). In order to further identify microbial taxa that serve as biomarkers for different groups, we performed liner discriminate analysis (LDA) coupled with effect size measurements (LEfSe). A cladogram (Fig. 2e) generated from LEfSe analysis showed the relationship between taxon and biomarker taxa (LDA score $>2$ and a significance of $P<0.05$ determined by the Wilcoxon signed-rank test) (Additional file 1: Figure S2b). Notably, in accordance with the SIMPER test, the LPS-producing phylum Proteobacteria and its members (class_y-Proteobacteria, family_Enterobacteriacea and genus_Escherichia/Shigella) were higher in the OVX group (Additional file 1: Figure S2b). We also demonstrated that a number of well-studied microbiota-based markers of obesity and MS associated with LGCI were affected by estrogen. These included the phylum Proteobacteria [14]; Firmicutes to Bacteroidetes ratio (FIR/BAC ratio-a known marker of obesity [14, 17]; Bifidobacterium/Enterobacteriaceae ratio (B/E) - a well-established marker of colonization resistance to opportunistic pathogens [27]; and the genus Akkermansia, which has been shown to reduce fat mass gain and WAT macrophage infiltration and improve gut barrier function and glucose metabolism [28]. Sexual dimorphism was clearly identified with respect to all four markers, with significantly lower Proteobacteria abundance, decreased FIR/BAC ratios, higher $\mathrm{B} / \mathrm{E}$ ratios, and increased Akkermansia abundance in normal females compared to normal male and OVX females (Fig. 2f-i). 17 $\beta$-E treatment significantly reduced Proteobacteria and elevated Akkermansia in the male (M+E2) (Fig. 2f, i) and significantly reduced the Proteobacteria and increased the $\mathrm{B} / \mathrm{E}$ ratio in OVX females $(\mathrm{OVX}+\mathrm{E} 2)$ (Fig. 2f, h). In addition, resulting taxa identified with pairwise comparison analyses at different taxonomic levels are summarized in the Additional file 1: Table S3. Next, we employed PICRUSt to predict the metagenomes and determine the changes in microbial metabolic pathways (KEGG) across different groups [29]. Male/OVX groups were segregated from the other three groups using PCoA applied on predicted functional pathways (Additional file 1: Figure S2c). According to LEfSe analysis, LPS-related functional pathways were significantly lower in the female, $\mathrm{M}+\mathrm{E} 2$ and $\mathrm{OVX}+\mathrm{E} 2$ groups compared to male and OVX groups (Additional file 1: Figure $S 2 d-f)$. The $\alpha$-diversity profile for groups reached stable values as indicated by the observed plateaus seen for each group in the Rarefaction curves (Additional file 1: Figure S3a-e). Together, these results highlight the sex specific shifts in gut microbiome that occurred upon WD and $17 \beta$-E treatment.

We next used fecal microbiota transplantation (FMT) and antibiotic (ABX)-induced depletion to investigate whether gut microbiota were necessary to mediate sexual dimorphism in MS. FMT with fecal content from donor male mice fed WD for 10 weeks was performed on female 10-week-old recipient mice pretreated with an antibiotic cocktail [19]. Unexpectedly, male mice fecal-transplant induced obesity (Fig. $3 \mathrm{a}-\mathrm{c}$ ) and glucose intolerance (Fig. 3d and Additional file 1: Figure S3f) and elevated serum TG (Fig. 3e), and markers of ME (Fig. 3f-h) and LGCI (Fig. 3i) in female recipients, with no differences between male and $\mathrm{M} \rightarrow \mathrm{F}$ groups $(n=5$ per group). Moreover, fecal $\beta$-diversity microbiota analysis (Fig. 3j) and $\mathrm{HCN}$ with heat-map analysis (Fig. 3k) showed that the $M$ and $M \rightarrow F$ samples were clustered together and were separated from the female control group ( $n=3$ per group). $\mathrm{M}$ and $\mathrm{M} \rightarrow \mathrm{F}$ groups presented with higher Proteobacteria and Firmicutes and lower Verrucomicrobia and Bacteroidetes compared to females (Fig. 3l). LEfSe showed $\gamma$-Proteobacteria and Enterobacteriacea for $\mathrm{M}$ and $\mathrm{M} \rightarrow \mathrm{F}$ groups analyzed as a single cluster (Fig. 3m and Additional file 1: Figure S3g). Higher RA of both Proteobacteria and FIR/BAC ratios, and lower $\mathrm{B} / \mathrm{E}$ ratio and Akkermansia muciniphila abundance (Additional file 1: Figure S2g-j) were also observed with the $\mathrm{M} \rightarrow \mathrm{F}$ group, while there were no significant differences in the $\alpha$-diversity measures (Additional file 1 : Figure S3h-l). Principal component analysis (PCA) with predicted metabolic pathways clustered the $\mathrm{M}$ and $\mathrm{M} \rightarrow$ F groups (Additional file 1: Figure S2k), and significantly higher abundance of LPS-related functions were found with LEfSe analysis (Additional file 1: Figure S2l). Finally, all of the abovementioned results were associated with undetectable $(<6.6 \mathrm{pg} / \mathrm{mL})$ serum E2 levels in the $\mathrm{M} \rightarrow$ F(Additional file 1: Figure S3m) group after FMT.

To further explore the causative role of gut microbiota, we next depleted the gut microbiota [14] in mice using a well-established ABX cocktail. Male and female mice ( $n=5$ /group) were fed WD for 10 weeks to induce MS. As expected, we found appreciable sexual dimorphism in the markers of ME (Fig. 4a), LGCI (Fig. 4b and Additional file 1: Figure S4a), and MS (Fig. 4c and Additional file 1: Figure S4b-d). Mice then received the ABX cocktail in drinking water for next 6 weeks. Strikingly, in addition to elimination of sexual dimorphism in markers of ME (Fig. 4a-d), LGCI (Fig. 4e-h and Additional file 1: Figure S4a), and MS (Fig. 4i-n and Additional file 1: Figure S4b-d), female mice showed significantly elevated levels of gut permeability (Fig. 4d), 

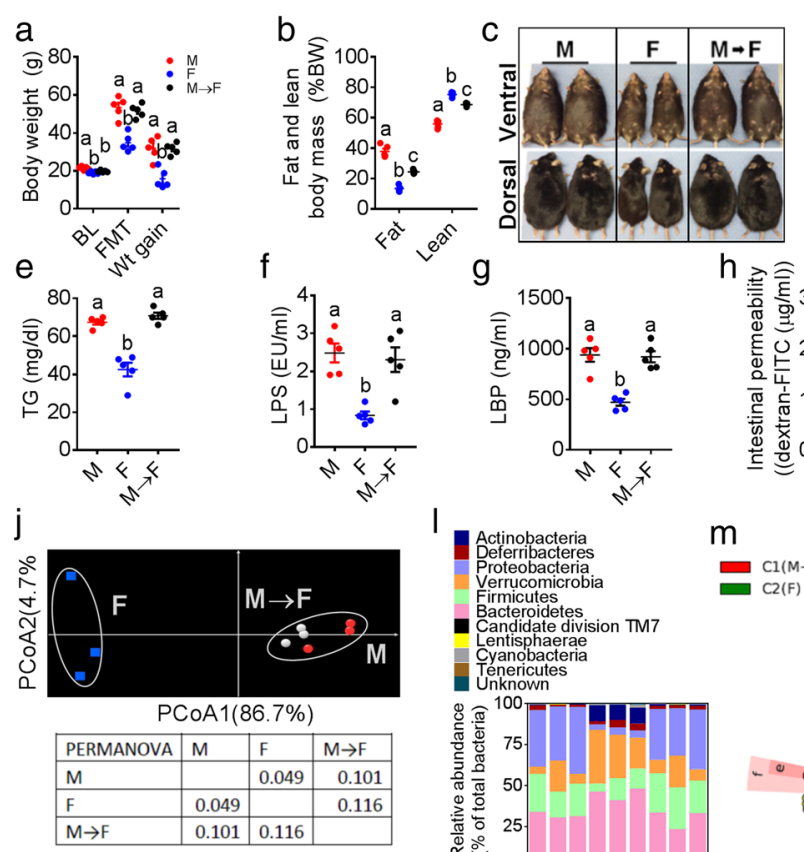

d
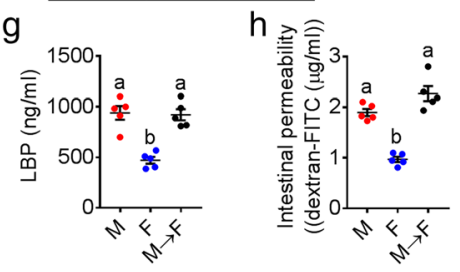

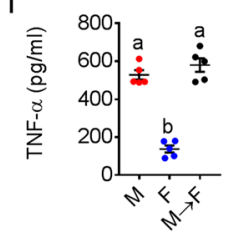
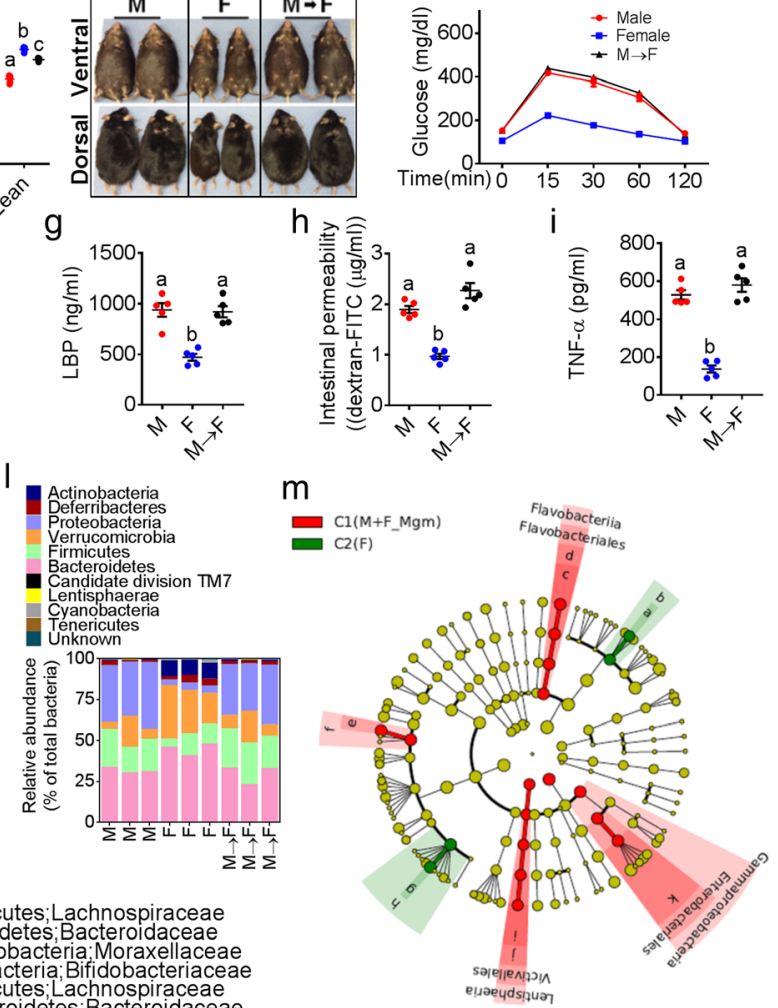

OTU-173; Firmicutes; Lachnospiraceae OTU-560; Proteobacteria: Moraxellaceae OU - Actinobacteria; Bitido bacteriaceae OTU-824: Bricutes, Lachnospiraceae OT-14: Bacteroidetes: Pocteryyromonadaceae
OTU-13:Bacteroidetes: Rikenellaceae OTU-290; Firmicutes:Lachnospiraceae OTU-251: Firmicutes; Erysipelotrichaceae ${ }^{100}$ OTU-150: Firmicutes: Ruminococcaceae OTU-21: Firmicutes; Ruminococcaceae OTU-315; Firmicutes; Lachnospiraceae OTU-606; Bacteroidetes: Bacteroidaceae -40 OTU-483:Bacteroidetes: Bacteroidaceae OTU- 23. Bacteroidetes Prevotellaceae OTU-132: Firmicutes:Family XII OIU-1110:Firmicutes:Ruminococcaceae OTU-273; Firmicutes; Ruminococcaceae OIU-214: Firmicutes; Lachnospiraceae OTU-1244; Proteobacteria; Enterobact OTU-634: Firmicutes;:Lachnospiraceae OTU-84:Bacteroidetes: Bacteroidaceae OTU-128; :irmicutes: Ruminococcaceae

Fig. 3 Male mice fecal microbiota-transplant transfers MS to female recipients. Fecal microbiota transplantation (FMT) with fecal content from donor male $(M ; n=5)$ mice fed WD was performed on female recipient mice $(F \rightarrow M ; n=5)$ pretreated with an antibiotic cocktail. Normal female $(F ; n=5)$ mice were maintained as a control group. a-e Markers of obesity (baseline (BL) and after FMT body weight and body weight gain in the end (a), fat and lean body mass (\% body weight) (b), gross appearance (dorsal and ventral aspects have been shown to differentiate the female from male sex) of male, female, and $\mathrm{F} \rightarrow \mathrm{M}$ mice $(\mathbf{c})$, glucose tolerance test results $(\mathbf{d})$, and serum triglyceride levels ${ }^{\oplus}$. $\mathbf{f}-\mathbf{h}$ Markers of metabolic endotoxemia such as serum lipopolysaccharide (LPS) concentration (f) and LPS-binding proteins (LBP) concentrations (g) and intestinal permeability to FITC-dextran macro molecules (h). i Serum TNF-a. $\mathbf{j} \beta$-diversity analysis using principal coordinate analysis (PCOA) with Bray-Curtis dissimilarity index followed by PERMANOVA significance test. $\mathbf{k}$ Hierarchical clustering with a heat map shows the representative OTU (those with greatest difference between groups) group means from each family selected for $P<0.05$, obtained with differential abundance analysis. I Phylumlevel analysis using the relative abundance (RA). $\mathbf{m}$ Cladogram generated from LEfSe analysis showing the relationship between biomarker taxa (the levels represent, from the inner to outer rings, phylum, class, order, family, and genus). ${ }^{\circledR D}$ Data was shown as mean \pm SEM. Data with different superscript letters are significantly different $(P<0.05)$. Ordinary or repeated measures one-way ANOVA followed by Tukey's multiple comparisons test

LGCI (Fig. 4e-h), body weight gain (Fig. 4i-j), glucose intolerance (Fig. 4k and Additional file 1: Figure b), HOMA-IR (Fig. 4i), and serum TG (Fig. 4m). Interestingly, female mice showed significantly lower serum E2 levels after $\mathrm{ABX}$ treatment compared to before $\mathrm{ABX}$ treatment (Additional file 1: Figure S4e). In combination, the results of these FMT and ABX experiments suggest that the gut microbiome mediates sexual dimorphism in the MS mainly through regulating the development ME and LGCI. 

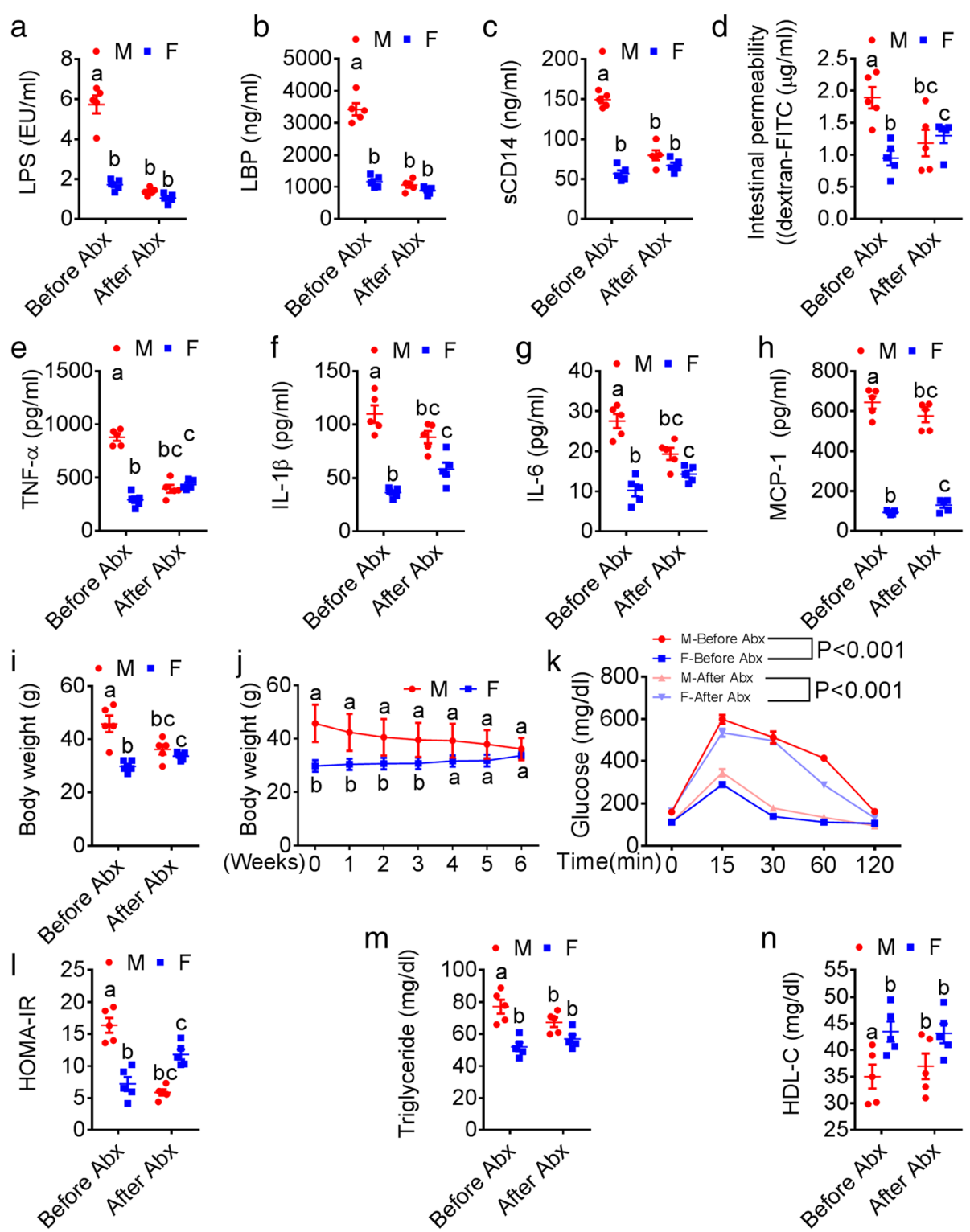

Fig. 4 Antibiotic treatment alleviates sexual dimorphism in MS. 10-week-old male $(n=5)$ and female $(n=5)$ mice maintained on chow diet were switched to a WD until the age of 20 weeks to induce sexual dimorphism in MS. Then, a broad-spectrum antibiotic cocktail (ABX) containing ampicillin (1 g/L), vancomycin (500 mg/L), neomycin sulfate (1 g/L) (added to drinking water), and metronidazole (100 mg/kg) (orally gavaged every $12 \mathrm{~h}$ ) was administered for next 6 weeks to deplete the gut microbiota. a-d Sexual dimorphism in markers of metabolic endotoxemia (serum LPS, LBP, sCD14, and intestinal permeability). e-h Markers of low-grade chronic inflammation (TNF-a, IL-1 $\beta$, IL-6, MCP-1, and IL-10). i-n Features of metabolic syndrome (body weight, glucose tolerance test, insulin resistance (HOMA-IR), serum triglycerides and HDL-C). Data (M vs. F and Before Abx vs. After Abx) were analyzed at the baseline (after 20 weeks of WD feeding) and after ABX treatment. Data was shown as mean \pm SEM. Data with different superscript letters are significantly different $(P<0.05)$. Ordinary or repeated measures two-way ANOVA followed by Sidak's multiple comparisons test

Isoflavones produce microbiome modifying effects similar to estrogen and reverse MS in male mice

To explore whether estrogen-like compounds can produce similar effects to those we found in the $17 \beta$-E-treated male, we used isoflavones (ISO) such as genistein (G) and daidzein (D). Male mice $(n=10)$ were fed WD for 4 months to induce severe obesity and MS and then treated with or without $\mathrm{G}$ and $\mathrm{D}$ isoflavones for 5 weeks. Interestingly, ISO treatment significantly reversed the WD-induced obesity (Fig. 5a-c), glucose intolerance and HOMA-IR (Fig. 5d-f), dyslipidemia (Fig. 5g-j), and NAFLD (including formation of Mallory bodies and mild fibrosis induced by WD) (Fig. 5k) despite similar energy intake between the male $(n=4)$ and male+ISO $(n=6)$ groups (Additional file 1 : Figure S5a). As expected, the reversal of MS parameters was associated with lower levels of markers of ME (Fig. 5l) 


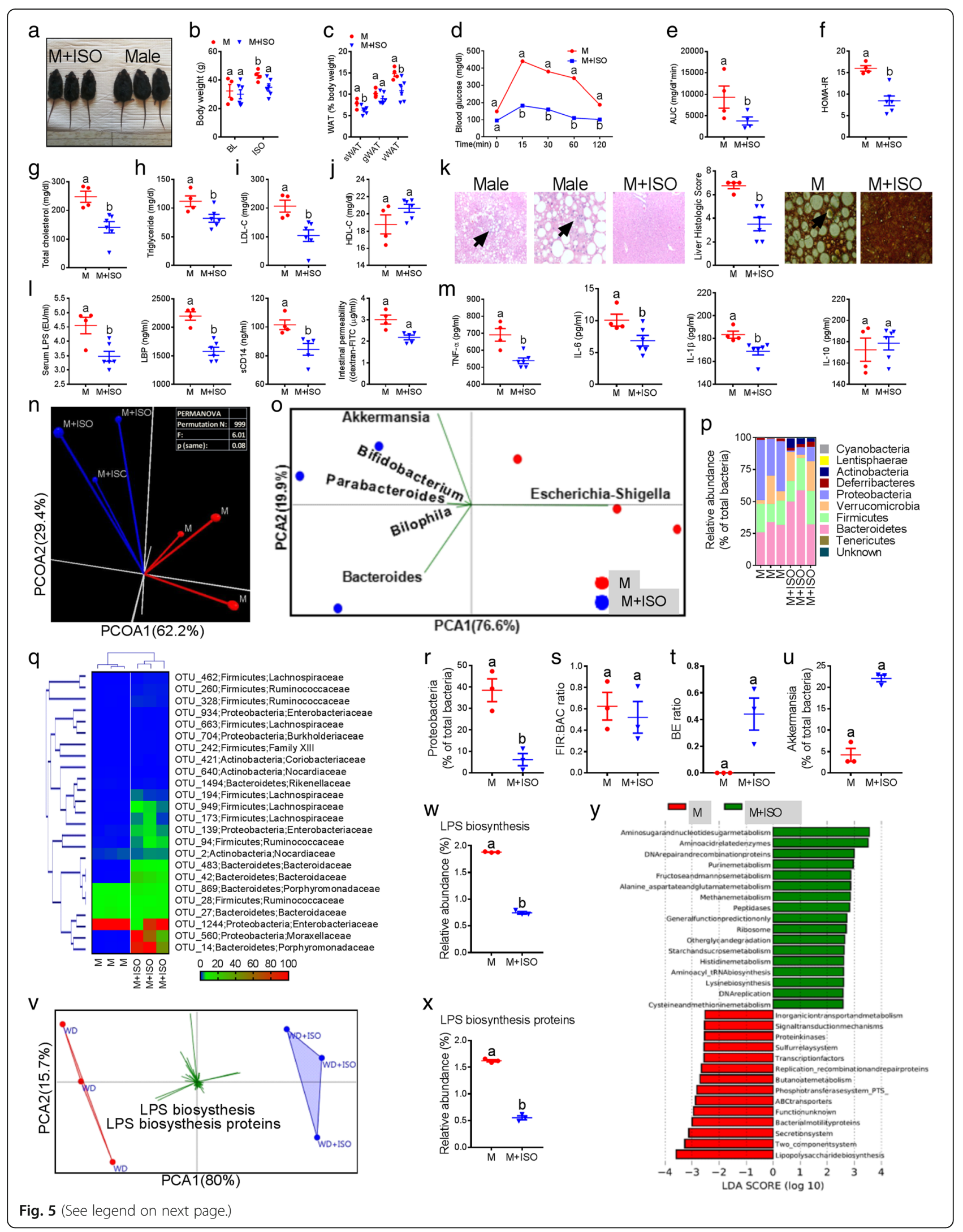




\begin{abstract}
(See figure on previous page.)
Fig. 5 Isoflavones produce similar microbiome-modifying effects as estrogens and reverse MS. Male mice with obesity and metabolic syndrome features were divided into Male $(n=4)$ and Male+ISO $(n=6)$ groups. Male+ISO group received supplementation of both genistein $(G)$ and daidzein with a WD. a-k Metabolic syndrome markers such as gross appearance and body weight (a-b) and white adipose tissue (WAT) weight (c), glucose tolerance test curves with area under the curve (AUC) (d, e) and insulin resistance (HOMA-IR) (f), serum total cholesterol, triglycerides, LDL-C and HDL-C ( $\mathbf{g}-\mathbf{j})$ histological evidences showing non-alcoholic fatty liver disease features (arrow heads indicating inflammatory cells infiltration, Mallory bodies and mild fibrosis) (k). I Markers of metabolic endotoxemia (serum LPS, LBP, sCD14, and intestinal permeability). $\mathbf{m}$ Markers of low-grade chronic inflammation (TNF-a, IL-6, IL-1 $\beta$, and IL-10). $\mathbf{n} \beta$-diversity analysis using principal coordinate analysis (PCOA) with Bray-Curtis dissimilarity index (BCD) followed by PERMANOVA significance test. o SIMPER with BCD analysis followed by principal component analysis (PCA) (variance-covariance type) showing the top six operational taxonomic unit (OTU) scores included as vectors. $\mathbf{p}$ Analysis at the phylum level using RA (\%). q Hierarchical clustering with a heat map shows the relative abundance (RA) of representative OTUs (those with greatest difference between groups) group means from each family selected for $P<0.05$, obtained with differential abundance analysis. $\mathbf{r}$-u Box plots showing the Proteobacteria, Firmicutes (FIR) to Bacteroidetes (BAC) ratio, Bifidobacterium (B) to Enterobacteriacea (E) ratio, and RA of genus Akkermansia. v RA of predicted microbial genes was identified using PICRUSt analysis followed by PCA (variance-covariance type) and the resulting scores were included as vectors. $\mathbf{w}, \mathbf{x}$ RA of lipopolysaccharide (LPS) biosynthesis and LPS biosynthesis proteins. $\mathbf{y}$ LDA scores derived from LEfSe analysis conducted on predicted microbial genes identified by PICRUSt, showing the biomarker functions. Data was shown as mean \pm SEM. Data with different superscript letters are significantly different $(P<0.05)$. Student's $t$ test or repeated measures one-way ANOVA followed by Tukey's multiple comparison test
\end{abstract}

and LGCI (Fig. 5m). Furthermore, we found higher mRNA levels of estrogen receptor (ER)- $\alpha$ and no changes with ER- $\beta$ in the duodenal tissues of ISO-treated male mice (Additional file 1: Figure S5b-c). Moreover, principal coordinate $\beta$-diversity analysis applied on entire fecal microbiome population distinctly clustered the Male+ISO and Male samples on axis one (Fig. 5n). Interestingly, similar to $17 \beta-E$, SIMPER test showed a higher contribution of Escherichia-Shigella to the M group whereas Akkermansia, Bifidobacterium, and Bacteroides were associated with the M+ISO group (Fig. 5o). At the phylum level, ISO treatment significantly reduced the RA of Proteobacteria and increased Actinobacteria and Verrucomicrobia (Fig. 5p). Differential expression with $\mathrm{HCN}$ analysis also grouped the $\mathrm{M}$ + ISO samples and the RA of major bacterial groups as shown in the heat map (Fig. 5q). In addition, significantly lower Proteobacteria levels (Fig. 5r), higher B/E ratios (Fig. 5t), increased Akkermansia levels (Fig. 5u), and significantly greater $\alpha$-diversity measures (Additional file 1: Figure S5b-f) were observed in the M+ISO group, although no differences were observed with FIR/BAC ratios (Fig. 5s). PCA of predicted functional metabolic pathways also segregated the $\mathrm{M}+\mathrm{ISO}$ group from the male samples (Fig. 5v). In addition, the RA of LPS-related functional pathways were significantly lower in M+ISO group compared to male group (Fig. $5 \mathrm{w}, \mathrm{x}$ ), which was also confirmed by LEfSe analysis (Fig. 5y). These data suggest that dietary supplementation of phytoestrogens such as ISO may exert effects similar to those of $17 \beta-E$ on the microbiome, ME, and LGCI in males to prevent MS.

\section{Intestinal alkaline phosphatase (IAP) drives sexual dimorphism in gut microbiota}

It has been well established that endogenous antimicrobial peptides (eAMP) (e.g., IAP and Reg3r) maintain normal gut homeostasis and regulate gut microbiota composition [14, 30]. Next, we examined the extent to which sexual dimorphism in gut microbiome was driven by differences in AMP levels between males and females, and whether estrogen status affected endogenous eAMP production. Among the eAMPs analyzed (Fig. 6a, b and Additional file 1: Figure S5i-j), we found significant differences only in IAP between groups based on sex and estrogen treatment (Fig. 6a, b). Recently, we and others have shown the gut microbiome-modifying effects of intestinal alkaline phosphatase (IAP), especially with respect to LPS-related and gram-positive bacterial groups [14, 31-33]. In addition to in vitro cell culture studies, duodenal tissues of the study groups (Fig. 2) were analyzed for IAP after 6 weeks of $17 \beta$-E treatment. We found upregulated mRNA expression levels of $A k p 3$ (a major IAP isozyme) (Fig. 6a), downregulated expression of the Akp6 IAP isozyme (Fig. 6b), higher villus-associated enterocytes expression of IAP with immunohistochemical staining (Fig. 6c), elevated IAP protein levels (Fig. 6d), and elevated IAP enzymatic activity in the duodenum (Fig. 6e) of female and 17 $\beta$-E-treated male and OVX groups compared to normal male and OVX mice. Moreover, the stimulation of differentiated $\mathrm{Caco} 2$ cells with $17 \beta-E(10 \mathrm{nM})$ significantly elevated IAP levels in a time-dependent manner (Fig. 6f). Similarly, elevated mRNA expression of $A k p 3$ (Fig. 6g, with no detection of expression of $A k p 6)$, higher immunochemical staining of IAP (Fig. 6h, i), elevated IAP protein levels (Fig. 6j), and elevated IAP activity (Fig. 6k) were observed in the duodenum of $\mathrm{M}+\mathrm{ISO}$ group compared to male group after 5 weeks of ISO treatment. In addition, in vitro stimulation of Caco2 cells with $25 \mu \mathrm{M}$ concentration of either genistein (G) or daidzein (D) or G+D combination also resulted in increased IAP protein levels (Fig. 6l). We also examined the effects of IAP inhibition using L-phenylalanine (L-phe), a specific non-competitive inhibitor [14, 32, 34, 35] of IAP. L-phe $(10 \mathrm{mM})$ pretreatment of Caco2 cells blocked the elevation of IAP protein 


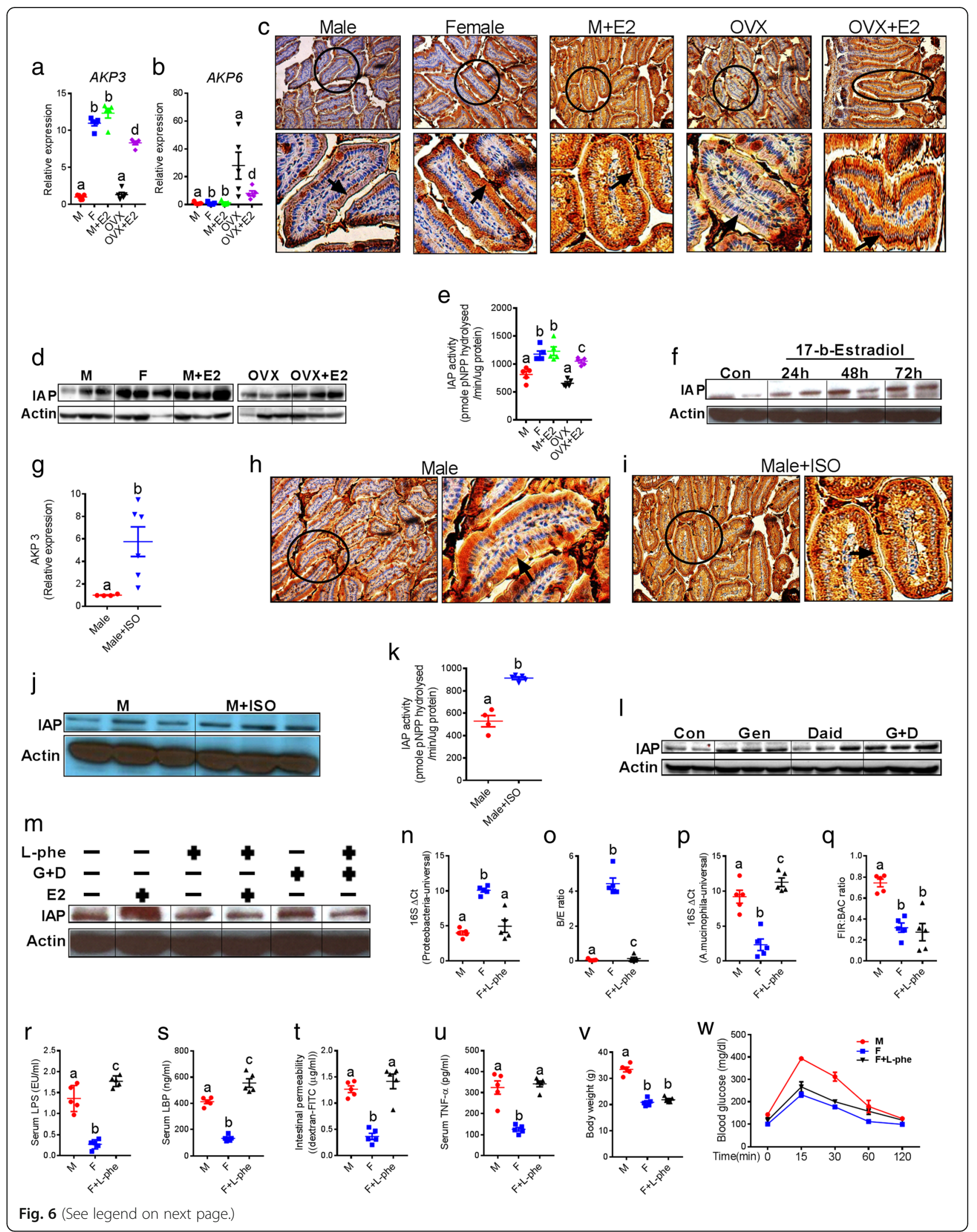


(See figure on previous page.)

Fig. 6 Intestinal alkaline phosphatase (IAP) mediates sexual dimorphism in gut microbiota in the context of MS. a, b mRNA expression of two major IAP isozymes AKP3 and AKP6 in the duodenum of five groups (male, female, male+E2, OVX, and OVX+E2; $n=5$ per group). c Immunohistochemical (IHC) localization of IAP in the duodenum. The enlarged view of single villus (circled in the main figures) has also been placed under each picture with an arrow head indicating the location of IAP in a circular fashion in the tip of small intestinal villus. $\mathbf{d}$ Western blot (WB) analysis showing the protein levels of IAP. e Duodenal tissue total IAP activity. $\mathbf{f}$ WB analysis of IAP using cell lysates collected from differentiated Caco-2 cell with enterocytes-like features after stimulating them (in vitro) with 17ß-estradiol (10 nM) in a time-dependent manner. g AKP3 mRNA expression of male (M) and male +ISO (M + ISO) groups. h, i Representative IHC staining pictures showing the IAP expression. $\mathbf{j}$ WB analysis showing the protein levels of IAP. $\mathbf{k}$ Total IAP activity. I In vitro stimulation of Caco-2 cells with $25 \mu \mathrm{M}$ concentration of either genistein (G) or daidzein (D) or G+D combination, followed by WB analysis of IAP. $\mathbf{m}$ In vitro stimulation of Caco-2 cells in the presence or absence of L-phenylalanine (10 mM), G+D (25 $\mu \mathrm{M})$, and $17 \beta$-E (10 nM), followed by WB analysis of IAP. q-PCR-based quantification of Proteobacteria, Bifidobacterium (B) to Enterobacteriacea (E) ratio and relative abundance of Akkermansia and Firmicutes (FIR) to Bacteroidetes (BAC) ratio $(\mathbf{n}-\mathbf{q})$, markers of metabolic endotoxemia (serum LPS, LBP and intestinal permeability) (r-t), low-grade chronic inflammation (TNF-a) (u), and markers of metabolic syndrome (body weight and GTT) (v, w) in the male (M), female (F), and $F+L-p h e(10 \mathrm{mM})$ treated groups. Data was shown as mean \pm SEM. Box plots (box showing the median, and the 25th and 75th percentiles, and the whiskers of the graph show the largest and smallest values) were also used. Data with different superscript letters are significantly different $(P<0.05)$. Student's $t$ test or ordinary or repeated measures one-way ANOVA followed by Tukey's multiple comparisons test

found with $17 \beta-E$ and ISO treatment (Fig. 6m). Further analysis using samples collected from in vivo (17 $\beta-E$ and ISO experiments in mice) and in vitro (Caco-2 cells) experiments indicated that both $17 \beta-\mathrm{E}$ and ISO significantly upregulated the mRNA levels of nuclear transcription factor KLF4 (gut-enriched Krüppel-like factor) (Additional file 1: Figure S5k-1). There was a clear trend of increased mRNA levels of Cdx1 (caudal-type homeobox-1) transcription factor (Additional file 1: Figure S5m-n), both KLF4 and Cdx1 are major transcription factors that target IAP [36]. To further verify whether IAP is one of the primary factors responsible for the differences in the gut microbiota between male and female C57BL/6 mice, we examined the effects of IAP inhibition on the status of key microbiota changes. After 8 weeks of WD and L-phe $(10 \mathrm{mM})$ treatment, we found that the L-phe-treated female mice exhibited a significant increase in LPS-producing Proteobacteria, decrease of $\mathrm{B} / \mathrm{E}$ ratio, increase of the relative abundance (RA) of Akkermansia muciniphila and no differences in FIR/BAC ratio (Fig. $6 \mathrm{n}-\mathrm{q}$ ) compared to the untreated female (F) group ( $n=5$ per group). Accordingly, the L-phe-treated female group showed significantly higher levels of markers of metabolic endotoxemia (Fig. 6r-t), LGCI (Fig. 6u), and glucose intolerance (Fig. 6v with no differences in body weight gain (Fig. 6w). These results support the notion that sexual dimorphism in endogenous IAP activity might partially drive differences in gut microbiota between male and female, and $17 \beta-E$ and ISO-treated C57BL/6 mice.

\section{Network interactions reveal host-microbiome interactions (HMI) driven by estrogen status}

Integrating significant microbial associations detected from OTU tables with metadata measurements of interest using correlation network analysis not only will provide us with valuable insights into the dynamics of the interactions between external factors and the microbial community, but also can help us understand how the detected relationships might change when additional variables are taken into account [37]. The HMI network (Fig. 7a) was first built from Spearman's nonparametric rank correlation coefficient $(P<0.05)$ between host parameters and microbial genera. Each node was colored according to the "data type" and sized based on "betweenness centrality," which quantifies the influence of a node in connecting other nodes within network values. Edges (lines) represent statistically significant correlations, and are colored light black for positive and blue for negative correlations. Next, nodes were grouped as modules and the first three largest modules of the network were taken to show the relationship between specific genera and host parameters (Fig. 7b, d). Partial least square (PLS) regression loading score plot (Fig. 7e) illustrates the association of between host parameters (dependent variables colored blue) and microbial genera (explanatory variables colored red). Samples from five different groups were observations (green dots). Accordingly, a diagram illustrating a proposed mechanism has been developed (Fig. 7f). Endogenous estrogens in the female mice, exogenous supplementation of $17 \beta-E$ to male and ovariectomized female mice, and dietary supplementation of ISO to male mice upregulate KLF4 transcription factor that target IAP, which, in turn, increases the endogenous IAP activity in the gut. Elevated IAP activity leads to decrease Proteobacteria and FIR/BAC, increase B/E, and the abundance of Akkermansia genera, decreasing LPS-producing bacteria (e.g., Proteobacteria) while increasing LPS-suppressing bacteria (e.g., Bifidobacterium and Akkermansia mucinophila). These changes lower LPS production and IP, resulting in reduced ME. The subsequent reduction of inflammatory cytokines leads to the suppression of LGCI and MS.

\section{Discussion}

To date, the mechanisms underlying the reported sexual dimorphism in MS have remained enigmatic. The present study demonstrates for the first time that sex-dependent 

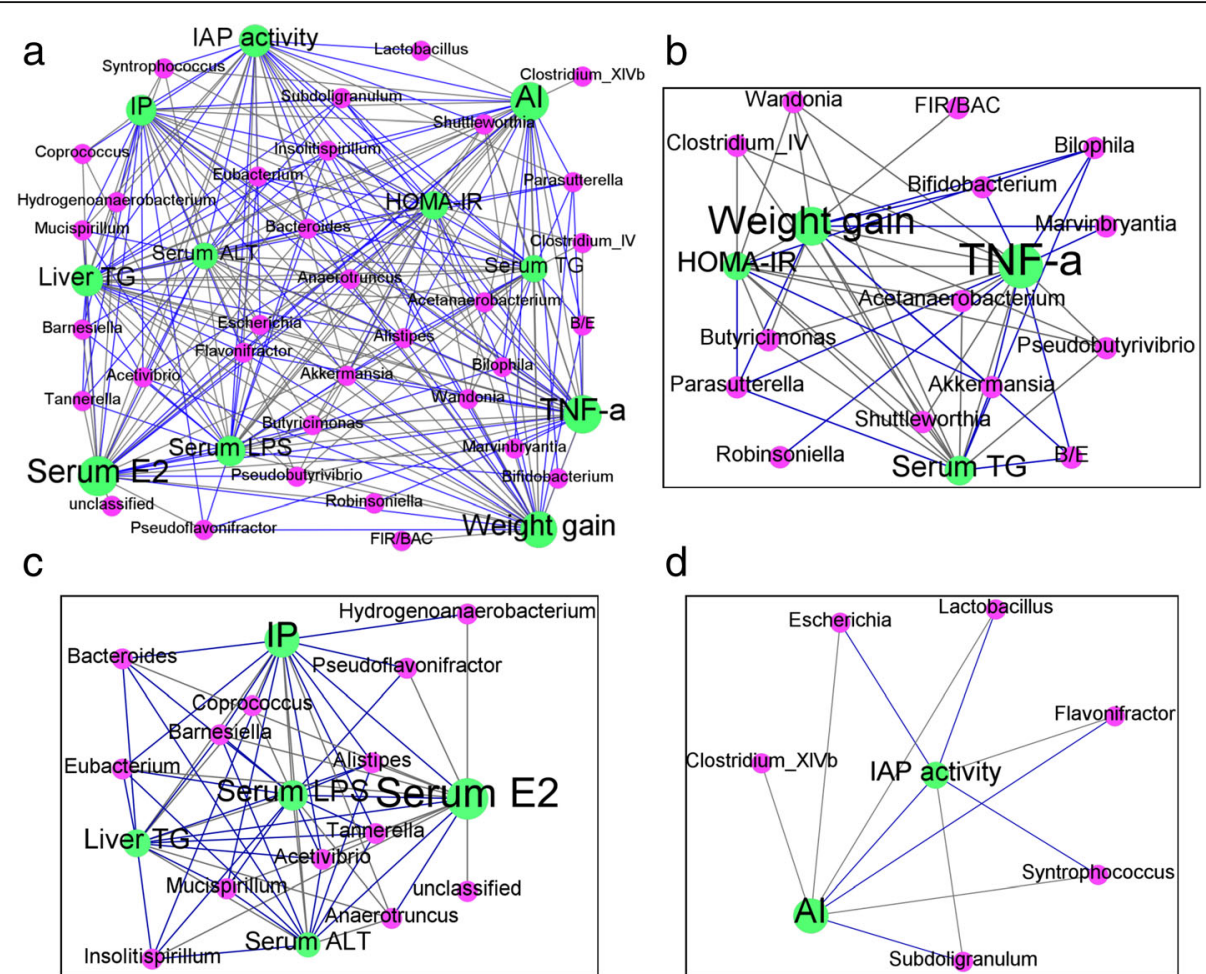

d
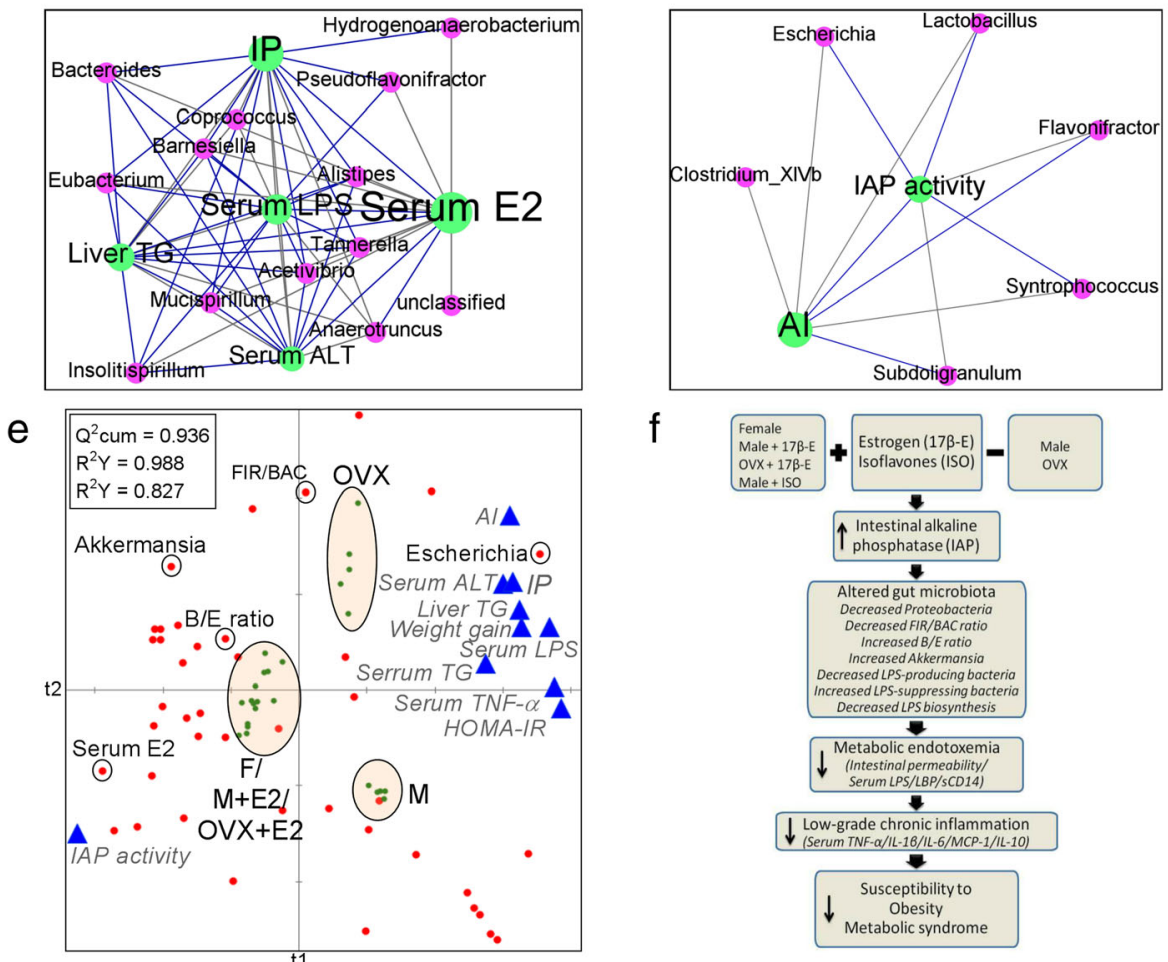

Fig. 7 Network interactions reveal host-microbiome interactions (HMI) driven by estrogen status. a-d The HMl network (a) was first built from Spearman's nonparametric rank correlation coefficient $(P<0.05)$ between host parameters and microbial genera. Each node was colored according to the "data type" and sized based on "betweenness centrality," which quantifies the influence of a node in connecting other nodes within network values. Edges (lines) represent statistically significant correlations and are colored light black for positive and blue for negative correlations. Next, nodes were grouped as modules (a set nodes connected to each other by many links, while connected by few links to nodes of other groups) and the first three largest modules of the network were taken to show the relationship between specific genera and host parameters (b-d). e Partial least square (PLS) regression loading score plot. The plot illustrates the association of between host parameters (dependent variables colored blue) and microbial genera (explanatory variables colored red). Samples from five different groups were observations (green dots). Leave one-out cross-validation (LOO-CV) was applied. The global goodness of fit $\left(Q^{2} c u m\right)$ and the predictive quality of the models $\left(R^{2} Y\right.$ and $R^{2} X$ cum) values were inserted on the top left corner of the figure. $\mathbf{f}$ Diagram illustrating a proposed mechanism. Endogenous estrogens in the female mice, exogenous supplementation of $17 \beta-$ estradiol (17ß-E) to male $(M+17 \beta-E)$ and ovariectomized female $(\mathrm{OVX}+17 \beta-E)$ mice and dietary supplementation of isoflavones (ISO) to male mice upregulate nuclear transcription factors (KLF4) that target IAP, which, in turn, increases the endogenous IAP activity in the gut. Elevated IAP activity leads to decreased Proteobacteria and Firmicutes to Bacteroidetes ratio (FIR/BAC), increased Bifidobacterium to Enterobacteriacea ratio (B/E), and the abundance of Akkermansia genera, decreasing LPS-producing bacteria (e.g., Proteobacteria) while increasing LPS-suppressing bacteria (e.g., Bifidobacterium and Akkermansia mucinophila). These changes lower LPS production (LPS biosynthesis and related proteins) and intestinal permeability, resulting in reduced metabolic endotoxemia (serum LPS/LPS binding proteins/soluble CD14). The subsequent reduction of inflammatory cytokines leads to the suppression of low-grade chronic inflammation and metabolic syndrome. Al, atherogenic index; TG, triglyceride; IAP, intestinal alkaline phosphatase; E2, $17 \beta$-estradiol 
effects on the gut microbiome mediate sexual dimorphism in MS in C57BL/6 mice and sex-specific expression and activity of IAP, a major gut microbiota-modifying factor [31-33]. Given the causative role of ME originating from dysbiotic gut microbiota and LGCI induced by ME in the context of WD-induced obesity and MS [14-16], the novel findings in our study are as follows: (1) sex-specific differences in the gut microbiota composition (e.g., Proteobacteria, FIR/BAC ratio, B/E ratio, and Akkermansia) and functions (e.g., LPS biosynthesis and LPS-related proteins) and markers of ME and LGCI induced by WD are associated with sexual dimorphism in MS. (2) Male mice are markedly susceptible to ME and LGCI and female mice are exclusively protected from ME and LGCI. (3) Gut microbiota, especially LPS-related bacteria, mediate the sexual dimorphism in MS, reflected by the fact that male mice microbiota transplants induced ME, LGCI, and MS in the female recipients and that ABX abolished the sexual dimorphism and worsened the MS markers in female mice. (4) $17 \beta-E$ induces gut microbiome changes, which is associated with lower susceptibility to WD-induced ME, LGCI, and MS in the male and OVX mice. Moreover, $17 \beta$-E-induced gut microbiome changes and protection against MS are associated with elevation of activity and expression of IAP. (5) Estrogen-like compounds (e.g., isoflavones) had gut microbiome-modifying effects similar to estrogens and prevented WD-induced ME, LGCI, and MS in male mice by elevating IAP, indicating that dietary supplementation of isoflavones could be a potential alternative to $17 \beta-E$ to treat men and postmenopausal women who are affected by obesity and MS. (6) IAP could largely mediate the $17 \beta-E$ and ISO-induced gut microbiome changes, and this might be due to the E2-mediated upregulation of transcription factors that target IAP such as KLF4 and CDX1.

It is important to note that the major gut microbiota findings (changes in Proteobacteria, FIR/BAC ratio, $\mathrm{B} / \mathrm{E}$ ratio, and Akkermansia) reported here regarding sexual dimorphism have been studied extensively in animals and also in the humans in the context of obesity and MS [17-19]. Data obtained from animal models identified consistent differences in the two major bacterial phyla, with a significant increase in Firmicutes and decrease in Bacteroidetes levels in genetically obese mice compared to wild-type mice despite similarities in their diet and activity levels [16]. Consistent with pre-clinical data, numerous human studies have consistently demonstrated that the FIR/BAC ratio is specifically increased in obese people [17-19]. Metabolic endotoxemia derived from gut dysbiosis is central to the pathogenesis of chronic low-grade inflammation, a factor underlying many current chronic diseases $[9,22]$. Metabolic endotoxemia can be determined by the abundance of bacteria affecting LPS production and gut barrier function. It is therefore conceivable that the $17 \beta$-E-induced marked reductions in LPS-producing bacteria (e.g., Proteobacteria) and increases in LPS-suppressing bacteria (e.g., Bifidobacterium and A. mucinophila) [14, 25-27] significantly suppressed the development of endotoxemia and inflammation. Recent clinical studies have shown that LPS-producing bacteria are abundant in obese subjects with type 2 diabetes [14, 16, 38-40]. It has also been shown that male mice fed a WD rich in milk fat and omega-6 fatty acids exhibit overgrowth of LPS-producing Proteobacteria and reduction of LPS-suppressing Bifidobacterium spp [27, 41, 42]. Along these lines, the WD diet used in the present study induced a dramatic increase in Proteobacteria and a decrease in Bifidobacterium in male and OVX mice (Fig. 3 and 5). In this context, decreasing the abundance of LPSproducing bacteria and increasing the LPS-suppressing bacteria may be a key mechanism for the reduction of metabolic endotoxemia. Another potential mechanism contributing to the reduction of serum LPS may be a decrease in gut permeability, due to the observed elevation of gut barrier-protecting bacteria such as Bifidobacterium $[14,43]$ by estrogen in our study.

In postmenopausal women and in female animal models, lower estrogen levels are associated with increased visceral adiposity [10] and estrogen replacement improves glucose-insulin homeostasis [44]. We have shown here for the first time the effects of E2 replacement on gut microbiota and metabolic endotoxemia in OVX mice, which mimics the postmenopausal state. There is growing evidence for a fundamental role of estrogen in the regulation of obesity and related metabolic disorders in males [11, 45], and recent data from rodent studies suggest that hepatic estrogen signaling has a key role in the prevention of high-fat diet-induced insulin resistance in males. However, it is not known whether estrogen treatment in males protects MS by modulating gut microbiota. Our novel results show that estrogen treatment in males is associated with the modulation of gut microbiota and improvement in ME and LGCI, which is associated with improvements in weight management and obesity-induced metabolic changes (Fig. 7), supporting the concept that estrogen plays an important role in the control of serum LPS levels by affecting LPS-related gut microbiota.

IAP is an endogenous antimicrobial peptide with numerous physiological functions [46, 47]. It is highly expressed in the small intestine, secreted from apical enterocytes into the lumen in microvilli vesicles, and travels to the large intestine [48]. IAP is known to inhibit the growth of E. coli and gram-negative bacteria by dephosphorylating LPS located in the outer membrane [32, 48-51]. IAP is also able to dephosphorylate ATP [33], 
which has been shown to reduce the survival of gram-positive bacteria (46), and to support the growth of gram-negative bacteria such as $E$. coli [52]. Oral IAP supplementation has also been shown to prevent $E$. coli overgrowth [53]. We recently found in our fat- 1 mice model (transgenic mice with elevated tissue n-6/n-3 fatty acid ratio) that elevated endogenous IAP activity and expression is associated with lower levels of LPS-producing bacteria and higher levels of LPS-suppressing bacteria [14]. Moreover, we found that inhibition of IAP by phenylalanine, a frequently used specific inhibitor of endogenous IAP activity [14, 15, 34, 35], caused ME, LGCI, and MS by increasing the growth of LPS-producing Proteobacteria and reducing the growth of LPS-suppressing Bifidobacterium spp in a mouse model of elevated endogenous IAP activity $[14,15]$. It is clear that IAP expression in the intestine is a critical determinant of the gut microbiota profile. Our findings indicate that IAP may be a primary factor partially mediating the effects of estrogen on gut microbiota because IAP inhibition in the female mice led to the development of ME, LGCI, and MS, associated with dramatic increase of Proteobacteria and reduction of the $\mathrm{B} / \mathrm{E}$ ratio and A. mucinophila abundance (Fig. 6n-q). The mechanism by which estrogen may modulate IAP expression is possibly due to its regulatory effect on the KLF4 transcription factor, which has been shown to target IAP [36].

Isoflavones (ISO) are non-steroidal compounds that can bind to both ER- $\alpha$ and ER- $\beta$ due to their ability to mimic the conformational structure of estradiol [54, $55]$, and thereby imitate the actions of estrogens on target tissues [56]. Isoflavones are found in many legumes and are particularly abundant in soy products. Genistein (G) and daidzein (D), two major soy isoflavone glucosides, are present at high concentrations in soybeans and soybean-derived products and are a major source of xenoestrogen exposure in both humans (e.g., soy-based formula for infants, tofu) and animals (most commercially available diets). G and D are widely used as dietary supplements in the USA for various presumed health benefits [57]. Ferguson et al. [58] administered a low-dose endotoxin (LPS $1 \mathrm{ng} / \mathrm{kg}$ ) to induce postprandial transient endotoxemia in young, healthy volunteers and found that subjects with a high-isoflavone diet were protected against inflammation-induced decline in insulin sensitivity. Meals high in fat, or fat and simple carbohydrates, are known to induce metabolic endotoxemia [59], as characterized by increased circulating markers of inflammation, and hypothesized to be linked to transient bacteremia due to reduced gut barrier function [59]. Most importantly, ISO has been shown to have E2 mimetic effects in preventing ovariectomy-induced metabolic dysfunctions [60], adipose deposition [61], hypertriglyceridemia, and hepatic status [62] in animal studies. Isoflavones have been shown to improve intestinal barrier integrity [63] and reduce colitis in animal models [64] potentially through modulation of the gut microbiome [65]. ISO may therefore confer protection against diet-induced metabolic dysfunction and reduce the development of insulin resistance in males and post-menopausal women, therefore supporting our proposal that ISO could be an effective alternative to E2 treatment.

Although we claim to explain the metabolic sexual dimorphism by effects of E2 through gut microbiota, we acknowledge that many other studies in the field showed that the major impact of estrogens on the metabolism are through estrogen receptor expression in metabolic tissues [66-69]. Clinical trials revealed that hormone replacement therapy (HRT) in postmenopausal women reduced the features of MS and inflammation [70]. Conversely, findings from Women's Health Initiative clinical trials (WHI-CT) [71] did not support use of HRT for chronic disease prevention [72]. However, the WHI-CT results were based on a group of women who were much older than those normally treated with HRT and who had other numerous risk factors [73]. Noticeably, there were very limited surveys performed addressing the effects on the metabolic syndrome components in postmenopausal women [74]. Early treatment with low-dosage HRT in healthy perimenopausal women was found to have beneficial effects on the components of metabolic syndrome and could decrease the risk of cardiovascular events [74] since the absolute risk of CVD events were markedly lower in younger, compared to older, women [71].

Although our results from FMT (Fig. 3) and antibiotics (Fig. 4) experiments contradict the protective effect of endogenous estrogen against obesity in female mice, it is well known that antibiotic usage [75] or gut dysbiosis [76] impacts estrogen metabolism mediated by microbiota Consequently, changes in circulating levels of estrogen (Additional file 1 Figure S3m and Additional file 1: Figure S4e) were found in female mice that received male microbiota transplants or antibiotic treatment. We hypothesize that estrogen-mediated gut microbiome changes may be the cause for sex differences in obesity and MS in this study. It is important to note the estrogen-mediated sex differences and the role of the microbiome have been linked in other disease conditions (e.g., autoimmunity) [77]. The bacterial changes reported here are similar to previous studies where ovariectomy or estrogen supplementation was performed. A study with OVX animals showed elevated Firmicutes to Bacteroidetes ratio [78] and Escherichia coli [79] compared to normal females. Likewise, it was shown that E2 supplementation elevated the relative abundance of Akkermansia and Bifidobacterium in the male and OVX mice 
respectively [80-82]. Estrogen inhibited the overgrowth of Proteobacteria and E. coli and decreased the levels of LBS and LBP under simulated microgravity [83]. In addition, E2 supplementation in male [84] or OVX mice [61] prevented obesity and MS, and genistein prevented obesity and metabolic dysfunction in the mice, which was similar to E2 supplementation in the same study $[60,61]$.

\section{Conclusions}

Our novel data demonstrate that the gut microbiome mediates sexual dimorphism in MS. Overall measures of correlation, pairwise correlations, and multivariate correlation analyses between the microbiota and host parameters that we performed provided novel insight into the host-microbiota system in the context of sexual dimorphism in WD-induced MS. Estrogen or estrogen-like compounds induced elevated IAP levels likely by upregulating the function of the KLF4 transcription factor that targets IAP and subsequent gut microbiome changes lower LPS production and gut permeability, resulting in reduced ME and systemic LGCI with subsequent reduction in the susceptibility to develop WD-induced MS in estrogen-treated males and post-menopausal women. Because exogenous estrogen administration to male causes deleterious effects (e.g., feminization and cardiac dysfunction) [85], compounds with estrogen-like activity (e.g., isoflavones and 17 $\alpha$-estradiol [85]) and non-feminizing effects may represent an alternative approach to the management of obesity and MS in males. Understanding the molecular basis of estrogen-mediated changes in IAP activity and gut microbiome may provide new approaches to the management of obesity-associated metabolic disease in men and menopausal women with estrogen deficiency, a condition that can last approximately 30 years of a woman's life [86].

\section{Methods}

\section{Animals and diets}

All the mice used in this study were wild-type (WT) on a C57BL/6 background and bred at the Massachusetts General Hospital (MGH) animal facility or purchased from Charles River Laboratories. Mice were housed in a biosafety level 2 room in hard top cages with two or three mice per cage. Mice were maintained in a temperature-controlled room $\left(22-24{ }^{\circ} \mathrm{C}\right)$ with a 12 -h light/12-h dark diurnal cycle and allowed for food and water ad libitum. Diets used in this study were either normal chow diet (CD) (Laboratory Rodent Diet 5001) from LabDiet or western diet (WD) (D12079B) from Research diets, Inc., NJ, USA. All animal procedures in this study were carried out in accordance with the guidelines approved by the MGH Subcommittee on Research Animal Care.

\section{Animal experiments}

Body weight and food intake were measured every week for all mice. All food was replaced weekly to avoid contamination. For collection of blood samples, mice were fasted for $6 \mathrm{~h}$ during the light phase period and blood was taken from the facial vein unless otherwise specified. After $6 \mathrm{~h}$ fasting, animals used in this study were euthanized by i.p. injection of pentobarbital $(200 \mathrm{mg} /$ $\mathrm{kg})$. Several experiments with special treatments are described below.

1) Determination of sexual dimorphism in metabolic endotoxemia, low-grade chronic inflammation, and metabolic syndrome: Male (M) WT $(n=11)$ and female (F) $(n=11)$ mice were weaned and switched to western diet (WD) until the age of 20 weeks to induce severe WD-induced obesity and MS. Both groups were subjected to analysis of markers of metabolic endotoxemia (ME) (including LPS, LBP, sCD14, and intestinal permeability), systemic lowgrade chronic inflammation (LGCI) (including TNF- $\alpha$, IL-1 $\beta$, IL-6, MCP-1, and IL-10), and metabolic syndrome (MS) (including weight gain, glucose tolerance test with area under the curve, insulin resistance index assessed by HOMA-IR, serum lipid profile including total cholesterol (TC), triglyceride (TG), low-density lipoprotein-C (LDL$\mathrm{C})$ and high-density lipoprotein-C (HDL-C) and atherogenic index, non-alcoholic fatty liver score and serum aspartate transaminase (AST) and alanine transaminase (ALT), and analysis of fecal microbiota by $16 \mathrm{~S}$ rRNA gene sequencing and serum LPS levels by LAL assay. The mice were then euthanized and white adipose tissue (WAT), which includes visceral (vWAT), subcutaneous (sWAT), and epididymal (eWAT) fat pads, and liver weights were taken. A portion of the liver was stored in 10\% formalin for histological analysis. Tissues were snap frozen in liquid nitrogen and then stored at $-80{ }^{\circ} \mathrm{C}$ for future analyses.

2) Determination of $17 \beta$-estradiol effects on gut microbiome, metabolic endotoxemia, low-grade chronic inflammation, and metabolic syndrome: Ten-weekold male (M), female (F), and ovariectomized (OVX) mice maintained on control diet were purchased from Charles River Laboratories and divided in to five groups ( $n=5 /$ group), and were fed WD and 17ß-estradiol (E2) from week 11 to week 17. The five groups are (1) M, (2) F, (3) M+E2, (4) OVX, and (5) OVX+E2. E2 (Sigma, USA) was prepared and given to groups 3 and 5 in the drinking water as described previously [87]. E2 was dissolved in $95 \%$ ethanol $(5 \mathrm{mg} / \mathrm{mL})$. Solubilized E2 was added to the drinking water to produce 
concentration of $4000 \mathrm{ng} \mathrm{E} 2 / \mathrm{mL}$ water with final ethanol concentration of $0.1 \%$. Groups 1,2 , and 4 received a normal drinking water bottle with $0.1 \%$ ethanol. The water bottles were changed every week for all the groups. After 6 weeks, mice were subjected to analysis for markers of ME and systemic LGCI and MS as mentioned in section 1 . The mice were then euthanized and WAT and liver weights were taken. A portion of the liver and duodenum was stored in 10\% formalin for histological analysis. Tissues were snap frozen in liquid nitrogen and then stored at $-80^{\circ} \mathrm{C}$ for future analyses.

3) Fecal microbiota transplantation (FMT): Mice from each group were individually housed for receiving antibiotic treatment to the end of the experiment. Fecal microbiota in place of cecal microbiota was transplanted for convenience and in order to minimize the number of animals used as donors. FMT with fecal content from donor male (M) mice that were fed WD for 10 weeks was performed on female 10-week-old mice [19]. Before the microbial transplantation, recipient mice $(\mathrm{M} \rightarrow \mathrm{F})$ were treated with a $200 \mu \mathrm{L}$ antibiotic cocktail (ampicillin, $1 \mathrm{~g} / \mathrm{l}$; metronidazole, $1 \mathrm{~g} / \mathrm{l}$; vancomycin, $0.5 \mathrm{~g} / \mathrm{l}$; neomycin, $0.5 \mathrm{~g} / \mathrm{l}$ ) (Sigma, USA) administrated by oral gavage once a day for 3 days. During the last $8 \mathrm{~h}$ of antibiotic treatment, mice were fed a WD to facilitate subsequent colonization. Fresh fecal pellets from donors were immediately weighed and placed into Ringer's solution and then diluted to $10 \mathrm{mg} /$ $\mathrm{mL}$. Immediately after diluting the fecal materials, fecal solutions were gavaged ( $200 \mu \mathrm{L}$ per mouse) to 4-h-fasted female recipients. Control groups of mice (males and females) were force-fed with $200 \mu \mathrm{L}$ of transfer buffer alone to eliminate the effects of gavage per se. Two days later, these mice received another gavage to exclude possibility of any unsuccessful inoculation. We conducted this FMT procedure once a week for the next 3 weeks. After the first microbiota gavage, all mice were fed a WD for 20 weeks. Measurement of body composition (fat mass) was performed using nuclear magnetic resonance (NMR) technique (minispec Body Composition Analyzer based on Time Domain NMR) that provides noninvasive and rapid measurement without anesthetics. Fecal samples from these three groups (M/F/M $\rightarrow$ F; $n=5$ per group) were collected after WD feeding for 20 weeks and three fecal samples from each group were subjected to $16 \mathrm{~S}$ rRNA sequencing.

4) Antibiotic $(A B X)$ treatment: 10 -week-old male $(n=$ $5)$ and female $(n=5)$ mice maintained on chow diet were switched to a WD until the age of 20 weeks to induce sexual dimorphism in MS. After collecting serum and stool for baseline measurements, both males and females receiving a WD started receiving a broad spectrum antibiotic cocktail (ABX) containing ampicillin $(1 \mathrm{~g} / \mathrm{l})$, vancomycin $(500 \mathrm{mg} / \mathrm{l})$, neomycin sulfate $(1 \mathrm{~g} / \mathrm{l})$ (added to the drinking water), and metronidazole $(100 \mathrm{mg} / \mathrm{kg})$ (orally gavaged every $12 \mathrm{~h}$ ) for 6 weeks to deplete the gut microbiota [88]. Validation of successful depletion of gut microbiota after the antibiotic treatment was performed as described previously [88]. Briefly, bacterial cultivation (both aerobic and anaerobic bacteria) of feces was performed on day 24 of the antibiotic treatment mice as described previously [14]. The detection limit of the assay (successful depletion) was defined as $1 \mathrm{cfu} / \mathrm{mg}$ feces. In addition, the bacterial genomic DNA was extracted from fresh stool samples of these mice and 16S rRNA gene copies for all bacteria (Table S4) was measured with qPCR method as described below. Both bacterial cultivation (cfu/mg feces) and qPCR results (Ct values) were compared with results from pre-antibiotic treatment fecal samples to confirm a significant depletion of gut microbiota. Mice with antibiotic treatment were subjected to analysis for markers of ME and systemic LGCI and MS as mentioned in section 1 .

5) Analysis of the effects of isoflavones (ISO) on gut microbiome, ME, LGCI, and MS: Ten-week-old male (M) mice were fed a WD for 4 months to induce MS and then divided into two groups: group 1: M, $(n=4)$, and group 2: M+ISO $(n=6)$. ISO such as genistein and daidzein (Cayman, USA) were supplemented at $0.1 \%$ in the WD, the M group was fed a WD, and the M+ISO group was fed a WD supplemented with ISO for the next 5 weeks. Mice were then subjected to analysis for markers of ME and systemic LGCI and MS as mentioned in section 1. The mice were then euthanized and white adipose tissue (WAT) and liver weights were taken. A portion of the liver and duodenum was stored in 10\% formalin for histological analysis. Tissues were snap frozen in liquid nitrogen and then stored at $-80^{\circ} \mathrm{C}$ for future analyses.

6) Analysis of the effects of intestinal alkaline phosphatase (IAP) inhibition on gut microbiome, ME, LGCI, and metabolic abnormalities: To determine the effects of inhibiting endogenous IAP activity, 12-week-old WT mice on a WD were divided into three groups ((male $(\mathrm{M})$, female $(\mathrm{F})$, and $\mathrm{F}+\mathrm{L}-\mathrm{phe})$ ) ( $n=5$ per group) and allowed to drink autoclaved water alone or water containing $10 \mathrm{mM}$ L-phenylalanine (L-phe) (Sigma, USA). After 8 weeks, the serum and fecal samples were collected to analyze the markers of ME, LGCI, and MS. 
Extraction of genomic DNA and profiling of the 16S rRNA gene by next generation Sequencing

Fecal DNA extraction and 16S rRNA gene sequencing Bacterial genomic DNA was extracted from fresh stool samples (100-180 mg) using the QIAamp DNA Stool Mini Kit (Qiagen, Valencia, CA), following the manufacturer's instructions. To increase effectiveness, the lysis temperature was increased to $95{ }^{\circ} \mathrm{C}$. Eluted DNA was treated with RNase and analyzed using a Nanodrop spectrophotometer (Biotek, Winooski, VT). Sample concentration and purity was determined by absorbance at $260 \mathrm{~nm}$ and the A260/A280 ratio, respectively. DNA samples packed with dry ice were shipped to APC Microbiome Institute (University College Cork, Cork, Ireland), and samples were sequenced as previously mentioned [37]. Briefly, V3-V4 amplicons for Illumina sequencing were generated according to the $16 \mathrm{~S}$ metagenomic sequencing library protocol (Illumina). An initial PCR reaction utilized primers specific for amplification of the $\mathrm{V} 3-\mathrm{V} 4$ region of the 16S rRNA gene, (Forward primer 5'TCGTCGGCA GCGTCAGATGTGTATAAGAGACAGCCTACGGGNG GCWGCAG; reverse primer 5' GTCTCGTGGGCTCG GAGATGTGTATAAGAGACAGGACTACHVGGGTATC TAATCC). PCR product clean-up and purification was achieved using the Agencourt AMPure XP system (Labplan, Dublin, Ireland). A second PCR incorporated a unique indexing primer pair for each sample (Illumina Nextera XT indexing primers, Illumina, Sweden). The products were again purified using the Agencourt AMPure XP system. Samples were quantified using the Qubit broad range DNA quantification assay kit (Bio-Sciences, Dublin, Ireland). Following quantification, samples were pooled in equimolar amounts $(4 \mathrm{nM})$ and sequenced at Clinical Microbiomcs, Copenhagen, Denmark, using Illumina MiSeq $2 \times 300$ bp paired end sequencing.

a)Bioinformatics Three hundred-base pair paired-end reads were assembled using FLASH with parameters of a minimum overlap of $20 \mathrm{bp}$ and a maximum overlap of 120 bp [89]. The QIIME suite of tools, v1.8.0, was used for further processing of paired-end reads, including quality filtering based on a quality score of $>25$ and removal of mismatched barcodes and sequences below length thresholds [90]. Denoising, chimera detection, and operational taxonomic unit (OTU) grouping were performed in QIIME using USEARCH v7 [91]. Taxonomic ranks were assigned by alignment of OTUs using PyNAST to the SILVA SSURef database release 111 [92]. Generation of $\alpha$ and $\beta$ diversities and analysis and visualization of principal coordinate analysis (PCoA) plots were performed using PAST and XLSTAT software. The $\alpha$-diversity of each group was calculated based on the annotated data using the diversity indices of the PAST version 2.17 software program [93]. Based on a non-parametric two-sample $t$-test using the default number of Monte Carlo permutations (999), comparative analyses of the group-specific $\alpha$-diversity indices were performed. Ordinations are the dimensional-reduction techniques which are commonly used to visualize complex relationships between communities between groups ( $\beta$-diversity). Dimensional reduction of the Bray-Curtis distance between microbiome samples using PCoA ordination method (PAST software) was done and significant differences among groups were tested with permutational multivariate analysis of variance (PERMANOVA), a multivariate non-parametric one-way ANOVA, which utilizes the sample-to-sample Bray-Curtis distance matrix directly. Taxa which were primarily responsible for an observed difference between groups were identified by SIMPER (similarity percentage analysis) method and their contribution to groups (between and within groups) were analyzed using the PCA variance-covariance type ordination (PAST software) method. Differential abundance analysis (non-parametric ANOVA with Benjamini-Hochberg FDRcorrected $P$ values $<0.05$ ) was performed on the RA of microbiota data at different levels of taxonomy to identify taxa with FDR-corrected $P$ values $<0.05$ (XLSTAT software; Addinsoft, USA) [94] and then their RA (normalized to percentage) were shown by a heat map with hierarchal clustering (HCN) analysis [95] using GraphPad Prism version 7.01 (La Jolla, CA). Linear discriminant analysis (LDA) effect size (LEfSe) is a biomarker discovery and explanation tool for high-dimensional data. It couples statistical significance with biological consistency and effect size estimation [96]. Microbiota-based biomarker discoveries were done with LEfSe using the online galaxy server (https://huttenhower.sph.harvard.edu/galaxy/), and the LDA scores derived from LEfSe analysis [96] were used to show the relationship between taxon using a cladogram (circular hierarchical tree) of significantly increased or decreased bacterial taxa in the gut microbiota between groups. Levels of the cladogram represent, from the inner to outer rings, phylum, class, order, family, and genus. Color codes indicate the groups, and letters indicate the taxa that contribute to the uniqueness of the corresponding groups at an LDA of $>2.0$. Unweighted pair-group method with arithmetic means (UPGMA) hierarchical clustering analysis diagram based on Bray-Curtis distance matrix was obtained using PAST version 3.11. Class trees were used to demonstrate similarity between samples, the clustering tree branch length was a measure of the cluster effect.

b)Putative metagenome identification Microbial functions were predicted using $16 \mathrm{~S}$ ribosomal RNA sequencing and phylogenetic reconstruction of unobserved 
states (PICRUSt) software (version 1.0.0) as described [29]. The predicted genes and functions were aligned to the KEGG database (version 66.1, May 1, 2013). PCA and PERMANOVA statistics were applied to check whether the groups were clustered according to predicted gene enrichments for microbial functions. LEfSe analysis [96] was utilized to determine significant putative KEGG orthologs and pathway analyses [96].

\section{Lipopolysaccharide (LPS) concentration}

Serum LPS concentrations were measured with a Toxin Sensor Chromogenic Limulus Amebocyte Lysate (LAL) Endotoxin Assay Kit (GenScript, Piscataway, NJ), following the manufacturer's instructions [15]. Briefly, serum samples were diluted 10- to 50-fold with endotoxin-free water, adjusted to the recommended $\mathrm{pH}$, and heated for $10 \mathrm{~min}$ at $70{ }^{\circ} \mathrm{C}$ to minimize inhibition or enhancement by contaminating proteins. LAL reagents were added to serum and incubated at $37{ }^{\circ} \mathrm{C}$ for $45 \mathrm{~min}$, and the absorbance was read at $545 \mathrm{~nm}$. All samples were validated for recovery and internal coefficient variation using known amounts of LPS.

\section{Intestinal permeability}

Intestinal permeability was determined as previously described [15]. Briefly, mice were gavaged with phosphate buffered saline (PBS, pH 7.2) containing $600 \mathrm{mg} / \mathrm{kg}$ body weight FITC-dextran (40 kDa, Sigma-Aldrich, USA). Blood samples $(120 \mu \mathrm{L})$ were collected after $90 \mathrm{~min}$. Serum was diluted with an equal volume of PBS, and fluorescence intensity was measured using a fluorospectrophotometer (excitation wavelength $480 \mathrm{~nm}$ and emission wavelength $520 \mathrm{~nm}$; Perkin-Elmer, Waltham, MA). Serum FITC-dextran concentrations were calculated from a standard curve of serially diluted FITC-dextran in PBS.

\section{Measurement of intestinal alkaline phosphatase (IAP) level and activity}

Small intestinal IAP specific activity (as it relates to protein) was measured as previously described [15] and expressed as picomoles pNPP hydrolyzed $/ \mathrm{min} / \mu \mathrm{g}$ of protein. Briefly, thoroughly washed duodenal tissues were homogenized with lysis buffer $(150 \mathrm{mM} \mathrm{NaCl}, 10 \mathrm{mM}$ Tris. $\mathrm{HCl}, \mathrm{pH} 7.5,1 \%$ sodium deoxycholate, $1 \%$ Nonidet P-40, $10 \mathrm{mM}$ EDTA, 0.1\% SDS, including protease inhibitor mixture; Sigma) followed by incubation on ice for $30 \mathrm{~min}$. Thereafter, the homogenates were centrifuged twice at $4{ }^{\circ} \mathrm{C}$ at $15,000 \mathrm{~g}$ for $15 \mathrm{~min}$, and the supernatants were collected to determine IAP activity as well as protein concentration. The Coomassie Blue Protein Assay (Bradford) kit from Fisher Scientific was used for protein quantification. For IAP assay, $25 \mu \mathrm{L}$ of supernatant was mixed with $175 \mu \mathrm{L}$ phosphatase assay reagent containing $5 \mathrm{mM}$ of p-nitrophenyl phosphate (pNPP) followed by determining optical density at $405 \mathrm{~nm}$. The specific activity of the enzyme was expressed as picomoles pNPP hydrolyzed $/ \mathrm{min} / \mu \mathrm{g}$ of protein. Protein concentration in a specific sample was determined using the protein assay reagents from Fisher Scientific.

\section{Cell culture experiments}

The human colon carcinoma cell line (Caco-2) was obtained from American Type Culture Collection (ATCC) (Rockville, MD) and cells were cultured in Dulbecco's modified Eagle's medium (DMEM) (Corning Inc., NY, USA) containing $4.5 \mathrm{~g} / \mathrm{l}$ glucose, $4 \mathrm{mmol} / \mathrm{l}$ L-glutamine, and $1 \mathrm{mmol} / \mathrm{l}$ sodium pyruvate, and supplemented with $10 \%$ fetal bovine serum (Cell Applications, Inc., San Diego, CA), $100 \mathrm{U} / \mathrm{mL}$ penicillin, and $100 \mu \mathrm{g} / \mathrm{mL}$ streptomycin (Gibco, NY, USA) in a humidified atmosphere of $5 \% \mathrm{CO}_{2}$ at $37{ }^{\circ} \mathrm{C}$. They were routinely subcultured when they were about $80 \%$ confluent. The culture medium was changed every other day. Cells were always $>90 \%$ viable, as shown by trypan blue (Invitrogen, Carlsbad, CA, USA) exclusion. Cells were passaged every 34 days by treatment with $0.1 \%$ trypsin (Gibco) and $0.04 \%$ ethylenediaminetetraacetic acid (EDTA) and then plated at a density of $1.3-2 \times 10^{4}$ cells $/ \mathrm{cm}^{2}$. Cells at passage number 17 were used for the experiments. All assays were done using only differentiated Caco-2. Cells were seeded in to six-well plates at $2 \times 10^{4}$ cells per well and treated with vehicle (ethanol or DMSO) or $10 \mathrm{nM}$ $17 \beta$-estradiol (E2) for 24,48 , and $72 \mathrm{~h}$ or each $25 \mu \mathrm{M}$ genistein $(\mathrm{G})$ or daidzein (D) or G+D mixture for $72 \mathrm{~h}$. In a subset of experiments, Caco-2 cells were pre-treated with L-phenylalanine $(10 \mathrm{mM})$ for $24 \mathrm{~h}$ and then they were treated with either vehicle or each $25 \mu \mathrm{M} \mathrm{G}+\mathrm{D}$ mixture or $10 \mathrm{nM}$ E2 for $72 \mathrm{~h}$. Medium was removed and cells were washed twice with ice-cold PBS, scraped, lysed in Trizol (Invitrogen), and stored at $-80{ }^{\circ} \mathrm{C}$ until mRNA was isolated or homogenized with $200 \mu \mathrm{L}$ radioimmunoprecipitation assay (RIPA) buffer (50 mM Tris- $\mathrm{HCl}, \mathrm{pH} 7.4,150 \mathrm{mM} \mathrm{NaCl}, 1 \%$ Triton $\mathrm{X}-100,1 \%$ sodium deoxycholate, $0.1 \%$ sodium dodecyl sulfate) containing protease inhibitor cocktail (Sigma), incubated on ice for $30 \mathrm{~min}$, centrifuged at $14,000 \mathrm{~g}$ for $10 \mathrm{~min}$ at $4{ }^{\circ} \mathrm{C}$ and the supernatant was collected and stored at $-80{ }^{\circ} \mathrm{C}$ for western blotting analysis.

\section{Western blotting analysis of IAP}

Western blotting on tissues and Caco-2 cell lysates was performed as previously described [14]. After thawed, the protein samples derived from Caco- 2 cells, the homogenates were centrifuged at $15,000 \mathrm{~g}$ at $4{ }^{\circ} \mathrm{C}$ for $15 \mathrm{~min}$, and the supernatants were collected. The duodenum section of small intestinal tissues was harvested and cut open longitudinally and luminal contents were 
removed. The tissues were washed with PBS and homogenized with liquid nitrogen, and homogenates were mixed with RIPA buffer, incubated on ice for $30 \mathrm{~min}$, and centrifuged at $14,000 \mathrm{~g}$ for $10 \mathrm{~min}$ at $4{ }^{\circ} \mathrm{C}$, and the supernatant was collected. Protein concentration of Caco- 2 and tissue homogenates was quantified by the Coomassie blue protein assay (Thermo Scientific, Rockford, IL, USA) using bovine serum albumin (BSA) as the standard. Proteins $(30 \mu \mathrm{g})$ were resolved on SDS-PAGE gels and transferred onto nitrocellulose membranes (Osmonics, Minnetonka, MN, USA). The membranes were blocked with $5 \%$ nonfat dry milk in Tris-buffered saline with $0.05 \%$ Tween 20 (TBS-T) for $1 \mathrm{~h}$ at room temperature and then probed with IAP primary antibodies (GTX27322, GeneTex, San Antonio, TX, USA) in $5 \%$ non-fat dry milk in TBS-T at $4{ }^{\circ} \mathrm{C}$ overnight. After washing three times in TBS-T, the blots were further incubated with the corresponding secondary antibodies conjugated with horseradish peroxidase for $1 \mathrm{~h}$ at room temperature (Santa Cruz Biotechnology, Santa Cruz, CA). Chemiluminescence was detected with Pierce ECL western blotting substrate (Thermo Scientific, Rockford, IL, USA) and visualized by ChemiDoc MP Imaging System (Bio-Rad, Hercules, CA, USA).

\section{Immunohistochemical analysis of IAP}

Formalin-fixed duodenal tissues and IAP primary antibodies (GTX27322, GeneTex) [14] were given to MGH core (Boston, MA). Prepared IHC slides were analyzed under light microscope and images of IAP staining and localization was taken by using $\times 20$ magnifications. All pictures were taken with the same exposure conditions without autoscaling.

\section{RV coefficient}

The RV coefficient was calculated between the microbial genera (FDR-corrected $P$ value $<0.05$ ) and the host parameters (markers of ME, LGCI and MS). The RV coefficient is a multivariate generalization of the Pearson correlation coefficient [97].

\section{Correlation network analysis}

Network-based analytical approaches have the potential to help disentangle complex host-microbe interactions [98]. Pairwise correlations between each microbiota (genera that are present at $<0.1 \%$ relative abundance in $>75 \%$ samples have been removed to avoid detecting spurious correlations among low-abundance OTUs) and host parameter (markers of ME, LGCI, and MS) were calculated using Spearman's nonparametric rank correlation coefficient [99]. Using those significant $(P<0.05)$ correlation coefficients, a correlation network (Fruchterman Reingold and label adjust layout) was built where nodes represent either a microbiota or a host parameter.
For each microbiota and a host parameter, an undirected edge was added between the corresponding nodes in the correlation network. Edges (light black links indicate positive and blue links indicate negative associations) represent statistically significant correlations $(P<0.05)$. Correlations were calculated using the PAST software version 2.17 and the network was visualized in Gephi Graph Visualization and Manipulation software version 0.9.2 [100]. Nodes were colored based on "data type" and sized based on "betweeness centrality (BC)." BC is a network centrality measure that quantifies the influence of a node in connecting other nodes in a network. It represents the fraction of all shortest paths in the network that pass through a given node. The nodes with the highest $\mathrm{BC}$ are usually known as highly central or hubs. A "module or component" in the network is a set of nodes connected to each other by many links, while connected by few links to nodes of other groups, so modules are elementary units of any biological network, and their identification and characterization provides us with more information about the local interaction patterns in the network and their contribution to the overall structure, connectivity, and function of the network. Modules are biologically important when considered as isolated, taxonomic, evolutionary, or functional modules. High modularity indicates that the network has dense connections within certain groups of nodes and sparse connections between these groups.

\section{Multivariate statistical analysis}

Partial least square regression (PLS-R) was used to associate the microbial composition to host parameters including jackknife-based variable selection [98]. PLS-R is recommended in cases of regression where the number of explanatory variables is high, and where it is likely that the explanatory variables are correlated. Leave one-out cross-validation (LOO-CV) was applied. The $\mathrm{Q}^{2}$ cumulated index $\left(\mathrm{Q} 2_{\text {cum }}\right)$ measures the global goodness of fit and the predictive quality of the models. Q2 $2_{\text {cum }}$ is also used to test the validity of the model against over-fitting. The cumulated $R^{2} Y$ and $R^{2} X$ cum that corresponds to the correlations between the explanatory $(\mathrm{X})$ and dependent $(\mathrm{Y})$ variables with the components are very close to one with two components in all the models. This indicates that the two components generated by the PLR-R summarize well both the Xs and the Ys. The results are also presented in PLS scatter plots for subject clustering and variables. The $R^{2}$ (coefficient of determination) indicates the $\%$ of variability of the dependent variable ( $\mathrm{Y}$ ) which is explained by the explanatory variables $(\mathrm{X})$. Parameters (variable importance in the projection values 1 or $>1.0$ ) contributing to the multivariate PLS models were compared with the corresponding identified modules (Fig. $7 \mathrm{~b}-\mathrm{d}$ ) in the 
correlation networks. All analyses were performed using precise algorithm in the XLSTAT software version 2017.6.

\section{Statistical analysis}

Data was shown as mean \pm standard error of mean (SEM). Box-plots (box showing the median, and the 25th and 75th percentiles, and the whiskers of the graph show the largest and smallest values) were also used to express the data. Unpaired Student's $t$ test was performed for experiments having only two groups. Either ordinary or repeated measures one-way or two-way analysis of variance (ANOVA) with Tukey's or Sidak's multiple comparisons post-test were used for experiments having more than two groups. If unequal variance was detected, data were analyzed using non-parametric tests. Differences were considered significant at $P<0.05$. Statistical analyses, including heat-map preparation, were performed using GraphPad Prism version 7.01 (GraphPad Software, La Jolla, CA). Differential expression analysis on $16 \mathrm{~S}$ sequencing data was conducted using XLSTAT software program [94]. Multivariate statistical analyses and power analyses (alpha $=0.05$; effect size $=$ 0.8) were conducted using PAST (version 2.17) [93] and XLSTAT (version 2017.6) software products.

\section{Additional file}

Additional file 1: Figure S1. Sexual dimorphism in the MS and ME and LGCl. Figure S2. Hierarchical clustering and predicted functional analysis. Figure S3. $\alpha$-and $\beta$ diversity analysis of fecal microbiota profile. Figure S4. Markers of low-grade inflammation and metabolic syndrome. Figure S5. Diversity analysis and antimicrobial peptides mRNA expression. Figure S6. The ingredients of Western diet. Table S1. Pairwise comparison of study groups with permutational multivariate analysis of variance. Table S2. Differential abundance analysis of fecal microbiota profile. Table S3. Pairwise differential abundance analysis between groups. Table S4. Primer sets used for real-time quantitative PCR. (DOC $13997 \mathrm{~kb}$ )

\section{Acknowledgements}

We thank Neil McKenna and Nicola Donelan (English language editors) for proofreading this manuscript. The authors are also grateful to Marina Kang for her editorial assistance and Jennifer Bian for her experimental assistance. The authors declare no competing financial interests.

\section{Funding}

This study was supported by generous funding from Sansun Life Sciences and the Fortune Education Foundation.

\section{Availability of data and materials}

OTU tables, raw data, taxonomy, FASTA files, scripts, PLS tables, and metadata for 165 rRNA gene sequence analysis performed in this study have been made publicly available in Figshare.

\section{Author's contributions}

JXK and KK conceived and designed the study; KK conducted all the mouse studies, collected serum and fecal and tissue samples, and performed biochemical and host-microbiota interaction analyses; RR, KM, and CS performed the 165 sequencing part of the study; CK, BW, and KK performed the predicted functional analysis using $16 \mathrm{~S}$ sequencing data; KK and JXK analyzed the metagenomic sequencing data; AKB performed the histopathological analyses of liver samples; $\mathrm{KK}$ and $\mathrm{LH}$ performed the cell culture experiments; KK and JXK wrote the manuscript; All authors approved the final version of the manuscript.

\section{Ethics approval}

All animal procedures in this study were performed in accordance with the ethical guidelines approved by the MGH Subcommittee on Research Animal Care.

\section{Competing interests}

The authors declare that they have no competing interests.

\section{Publisher's Note}

Springer Nature remains neutral with regard to jurisdictional claims in published maps and institutional affiliations.

\section{Author details}

'Laboratory of Lipid Medicine and Technology, Department of Medicine, Massachusetts General Hospital and Harvard Medical School, 149 -13th Street, Boston, MA 02129, USA. ${ }^{2}$ School of Microbiology, University College Cork, Cork, Ireland. ${ }^{3}$ Teagasc Moorepark Food Research Centre, Fermoy, Co. Cork, Ireland. ${ }^{4}$ APC Microbiome Institute, University College Cork, Cork, Ireland. ${ }^{5}$ Research Center for Nutrition and Food Safety, Institute of Military Preventive Medicine, Third Military Medical University, Chongqing Key Laboratory of Nutrition and Food Safety, Chongqing Medical Nutrition Research Center, Chongqing, People's Republic of China. ${ }^{6}$ Department of Pathology, Massachusetts General Hospital and Harvard Medical School, Boston, MA 02114, USA.

Received: 23 June 2018 Accepted: 30 October 2018

Published online: 13 November 2018

\section{k) References}

1. Cornier MA, Dabelea D, Hernandez TL, Lindstrom RC, Steig AJ, Stob NR, Van Pelt RE, Wang H, Eckel RH. The metabolic syndrome. Endocr Rev. 2008;29(7): 777-822.

2. Lutsey PL, Steffen LM, Stevens J. Dietary intake and the development of the metabolic syndrome: the atherosclerosis risk in communities study. Circulation. 2008;117(6):754-61.

3. Franconi F, Seghieri G, Canu S, Straface E, Campesi I, Malorni W. Are the available experimental models of type 2 diabetes appropriate for a gender perspective? Pharmacol Res. 2008:57(1):6-18.

4. Hwang LL, Wang CH, Li TL, Chang SD, Lin LC, Chen CP, Chen CT, Liang KC, Ho IK, Yang WS, et al. Sex differences in high-fat diet-induced obesity, metabolic alterations and learning, and synaptic plasticity deficits in mice. Obesity (Silver Spring). 2010;18(3):463-9.

5. Corsetti JP, Sparks JD, Peterson RG, Smith RL, Sparks CE. Effect of dietary fat on the development of non-insulin dependent diabetes mellitus in obese Zucker diabetic fatty male and female rats. Atherosclerosis. 2000;148(2):231-41.

6. Regitz-Zagrosek V, Lehmkuhl E, Weickert MO. Gender differences in the metabolic syndrome and their role for cardiovascular disease. Clin Res Cardiol. 2006;95(3):136-47.

7. Giles DA, Moreno-Fernandez ME, Stankiewicz TE, Graspeuntner S, Cappelletti M, Wu D, Mukherjee R, Chan CC, Lawson MJ, Klarquist J, et al. Thermoneutral housing exacerbates nonalcoholic fatty liver disease in mice and allows for sex-independent disease modeling. Nat Med. 2017;23(7):829-38.

8. Guarner-Lans V, Rubio-Ruiz ME, Perez-Torres I, Banos de MacCarthy G. Relation of aging and sex hormones to metabolic syndrome and cardiovascular disease. Exp Gerontol. 2011;46(7):517-23.

9. Wajchenberg BL. Subcutaneous and visceral adipose tissue: their relation to the metabolic syndrome. Endocr Rev. 2000;21(6):697-738.

10. Carr MC. The emergence of the metabolic syndrome with menopause. J Clin Endocrinol Metab. 2003;88(6):2404-11.

11. Maffei L, Murata Y, Rochira V, Tubert G, Aranda C, Vazquez M, Clyne CD, Davis S, Simpson ER, Carani C. Dysmetabolic syndrome in a man with a novel mutation of the aromatase gene: effects of testosterone, alendronate, and estradiol treatment. J Clin Endocrinol Metab. 2004;89(1):61-70.

12. Dethlefsen $L, M c F a l l-N g a i$ M, Relman DA. An ecological and evolutionary perspective on human-microbe mutualism and disease. Nature. 2007; 449(7164):811-8.

13. Backhed F, Ding H, Wang T, Hooper LV, Koh GY, Nagy A, Semenkovich CF, Gordon $\mathrm{Jl}$. The gut microbiota as an environmental factor that regulates fat storage. Proc Natl Acad Sci U S A. 2004;101(44):15718-23.

14. Kaliannan K, Wang B, Li XY, Kim KJ, Kang JX. A host-microbiome interaction mediates the opposing effects of omega- 6 and omega-3 fatty acids on metabolic endotoxemia. Sci Rep. 2015;5:11276. 
15. Kaliannan K, Hamarneh SR, Economopoulos KP, Alam SN, Moaven O, Patel P, Malo NS, Ray M, Abtahi SM, Muhammad N, et al. Intestinal alkaline phosphatase prevents metabolic syndrome in mice. Proc Natl Acad Sci U S A. 2013;110(17):7003-8.

16. Chang CJ, Lin CS, Lu CC, Martel J, Ko YF, Ojcius DM, Tseng SF, Wu TR, Chen YY, Young JD, et al. Ganoderma lucidum reduces obesity in mice by modulating the composition of the gut microbiota. Nat Commun. 2015;6:7489.

17. Turnbaugh PJ, Ley RE, Mahowald MA, Magrini V, Mardis ER, Gordon JI. An obesity-associated gut microbiome with increased capacity for energy harvest. Nature. 2006:444(7122):1027-31.

18. Koliada A, Syzenko G, Moseiko V, Budovska L, Puchkov K, Perederiy V, Gavalko Y, Dorofeyev A, Romanenko M, Tkach S, et al. Association between body mass index and Firmicutes/Bacteroidetes ratio in an adult Ukrainian population. BMC Microbiol. 2017;17(1):120.

19. Caesar R, Tremaroli V, Kovatcheva-Datchary P, Cani PD, Backhed F. Crosstalk between gut microbiota and dietary lipids aggravates WAT inflammation through TLR signaling. Cell Metab. 2015;22(4):658-68.

20. Cani PD, Possemiers S, Van de Wiele T, Guiot Y, Everard A, Rottier O, Geurts $L$, Naslain D, Neyrinck A, Lambert DM, et al. Changes in gut microbiota control inflammation in obese mice through a mechanism involving GLP-2driven improvement of gut permeability. Gut. 2009;58(8):1091-103.

21. Cani PD, Bibiloni R, Knauf C, Waget A, Neyrinck AM, Delzenne NM, Burcelin R. Changes in gut microbiota control metabolic endotoxemia-induced inflammation in high-fat diet-induced obesity and diabetes in mice. Diabetes. 2008;57(6):1470-81.

22. Cani PD, Amar J, Iglesias MA, Poggi M, Knauf C, Bastelica D, Neyrinck AM, Fava F, Tuohy KM, Chabo C, et al. Metabolic endotoxemia initiates obesity and insulin resistance. Diabetes. 2007:56(7):1761-72.

23. Wellen KE, Hotamisligil GS. Inflammation, stress, and diabetes. J Clin Invest. 2005;115(5):1111-9.

24. Shi H, Kokoeva MV, Inouye K, Tzameli I, Yin H, Flier JS. TLR4 links innate immunity and fatty acid-induced insulin resistance. J Clin Invest. 2006; 116(11):3015-25.

25. Li J, Lin S, Vanhoutte PM, Woo CW, Xu A. Akkermansia Muciniphila protects against atherosclerosis by preventing metabolic Endotoxemia-induced inflammation in Apoe-/- mice. Circulation. 2016;133(24):2434-46.

26. Cani PD, Neyrinck AM, Fava F, Knauf C, Burcelin RG, Tuohy KM, Gibson GR, Delzenne NM. Selective increases of bifidobacteria in gut microflora improve high-fat-diet-induced diabetes in mice through a mechanism associated with endotoxaemia. Diabetologia. 2007;50(11):2374-83.

27. Kaliannan K, Wang B, Li XY, Bhan AK, Kang JX. Omega-3 fatty acids prevent early-life antibiotic exposure-induced gut microbiota dysbiosis and later-life obesity. Int J Obes. 2016;40(6):1039-42.

28. Everard A, Belzer C, Geurts L, Ouwerkerk JP, Druart C, Bindels LB, Guiot Y, Derrien M, Muccioli GG, Delzenne NM, et al. Cross-talk between Akkermansia muciniphila and intestinal epithelium controls diet-induced obesity. Proc Natl Acad Sci U S A. 2013;110(22):9066-71.

29. Langille MG, Zaneveld J, Caporaso JG, McDonald D, Knights D, Reyes JA, Clemente JC, Burkepile DE, Vega Thurber RL, Knight R, et al. Predictive functional profiling of microbial communities using $16 \mathrm{~S}$ rRNA marker gene sequences. Nat Biotechnol. 2013;31(9):814-21.

30. Ostaff MJ, Stange EF, Wehkamp J. Antimicrobial peptides and gut microbiota in homeostasis and pathology. EMBO Mol Med. 2013;5(10):1465-83.

31. Malo MS, Alam SN, Mostafa G, Zeller SJ, Johnson PV, Mohammad N, Chen KT, Moss AK, Ramasamy S, Faruqui A, et al. Intestinal alkaline phosphatase preserves the normal homeostasis of gut microbiota. Gut. 2010;59(11):1476-84.

32. Campbell EL, MacManus CF, Kominsky DJ, Keely S, Glover LE, Bowers BE, Scully M, Bruyninckx WJ, Colgan SP. Resolvin E1-induced intestinal alkaline phosphatase promotes resolution of inflammation through LPS detoxification. Proc Natl Acad Sci U S A. 2010;107(32):14298-303.

33. Malo MS, Moaven O, Muhammad N, Biswas B, Alam SN, Economopoulos KP Gul SS, Hamarneh SR, Malo NS, Teshager A, et al. Intestinal alkaline phosphatase promotes gut bacterial growth by reducing the concentration of luminal nucleotide triphosphates. Am J Physiol Gastrointest Liver Physiol. 2014;306(10):G826-38.

34. Li LN, Wu YQ, Buchet R. Kinetic study on inhibition effects of dansyl-Lphenylalanine and L-phenylalanine on calf intestinal alkaline phosphatase. Guang Pu Xue Yu Guang Pu Fen Xi. 2009;29(10):2820-3.

35. Ghosh NK, Fishman WH. On the mechanism of inhibition of intestinal alkaline phosphatase by L-phenylalanine. I Kinetic studies. J Biol Chem. 1966; 241(11):2516-22.
36. Lalles JP, Orozco-Solis R, Bolanos-Jimenez F, de Coppet P, Le Drean G, Segain JP. Perinatal undernutrition alters intestinal alkaline phosphatase and its main transcription factors KLF4 and Cdx1 in adult offspring fed a high-fat diet. J Nutr Biochem. 2012;23(11):1490-7.

37. Robertson RC, Kaliannan K, Strain CR, Ross RP, Stanton C, Kang JX. Maternal omega-3 fatty acids regulate offspring obesity through persistent modulation of gut microbiota. Microbiome. 2018;6(1):95.

38. de La Serre CB, Ellis CL, Lee J, Hartman AL, Rutledge JC, Raybould HE. Propensity to high-fat diet-induced obesity in rats is associated with changes in the gut microbiota and gut inflammation. Am J Physiol Gastrointest Liver Physiol. 2010;299(2):G440-8.

39. Sanz Y, Santacruz A, Gauffin P. Gut microbiota in obesity and metabolic disorders. Proc Nutr Soc. 2010;69(3):434-41.

40. Zhang C, Zhang M, Wang S, Han R, Cao Y, Hua W, Mao Y, Zhang X, Pang X, Wei $C$, et al. Interactions between gut microbiota, host genetics and diet relevant to development of metabolic syndromes in mice. ISME J. 2010;4(2):232-41.

41. Agus A, Denizot J, Thevenot J, Martinez-Medina M, Massier S, Sauvanet P, Bernalier-Donadille A, Denis S, Hofman P, Bonnet R, et al. Western diet induces a shift in microbiota composition enhancing susceptibility to adherent-Invasive E. coli infection and intestinal inflammation. Sci Rep. 2016;6:19032.

42. Huang EY, Leone VA, Devkota S, Wang Y, Brady MJ, Chang EB. Composition of dietary fat source shapes gut microbiota architecture and alters host inflammatory mediators in mouse adipose tissue. JPEN J Parenter Enteral Nutr. 2013;37(6):746-54.

43. Moya-Perez A, Neef A, Sanz Y. Bifidobacterium pseudocatenulatum CECT 7765 reduces obesity-associated inflammation by restoring the lymphocytemacrophage balance and gut microbiota structure in high-fat diet-fed mice PLoS One. 2015;10(7):e0126976.

44. Nabulsi AA, Folsom AR, White A, Patsch W, Heiss G, Wu KK, Szklo M. Association of hormone-replacement therapy with various cardiovascular risk factors in postmenopausal women. The atherosclerosis risk in communities study investigators. N Engl J Med. 1993;328(15):1069-75.

45. Finkelstein JS, Lee H, Burnett-Bowie SA, Pallais JC, Yu EW, Borges LF, Jones BF, Barry CV, Wulczyn KE, Thomas BJ, et al. Gonadal steroids and body composition, strength, and sexual function in men. N Engl J Med. 2013; 369(11):1011-22.

46. Lalles JP. Intestinal alkaline phosphatase: multiple biological roles in maintenance of intestinal homeostasis and modulation by diet. Nutr Rev. 2010;68(6):323-32

47. Lalles JP. Intestinal alkaline phosphatase: novel functions and protective effects. Nutr Rev. 2014;72(2):82-94.

48. Shifrin DA Jr, McConnell RE, Nambiar R, Higginbotham JN, Coffey RJ, Tyska MJ. Enterocyte microvillus-derived vesicles detoxify bacterial products and regulate epithelial-microbial interactions. Curr Biol. 2012;22(7):627-31.

49. Koyama I, Matsunaga T, Harada T, Hokari S, Komoda T. Alkaline phosphatases reduce toxicity of lipopolysaccharides in vivo and in vitro through dephosphorylation. Clin Biochem. 2002;35(6):455-61.

50. Chen H, Wang W, Degroote J, Possemiers S, Chen D, De Smet S, Michiels J. Arabinoxylan in wheat is more responsible than cellulose for promoting intestinal barrier function in weaned male piglets. J Nutr. 2015;145(1):51-8.

51. Yang C, Zhu X, Liu N, Chen Y, Gan H, Troy FA 2nd, Wang B. Lactoferrin upregulates intestinal gene expression of brain-derived neurotrophic factors BDNF, UCHL1 and alkaline phosphatase activity to alleviate early weaning diarrhea in postnatal piglets. J Nutr Biochem. 2014;25(8):834-42.

52. Mempin R, Tran H, Chen C, Gong H, Kim Ho K, Lu S. Release of extracellular ATP by bacteria during growth. BMC Microbiol. 2013;13:301.

53. De Lisle RC, Mueller R, Boyd M. Impaired mucosal barrier function in the small intestine of the cystic fibrosis mouse. J Pediatr Gastroenterol Nutr. 2011;53(4):371-9.

54. Kuiper GG, Lemmen JG, Carlsson B, Corton JC, Safe SH, van der Saag PT, van der Burg B, Gustafsson JA. Interaction of estrogenic chemicals and phytoestrogens with estrogen receptor beta. Endocrinology. 1998;139(10): 4252-63.

55. Al-Nakkash L. Genistein stimulates jejunal chloride secretion via sexdependent, estrogen receptor or adenylate cyclase mechanisms. Cell Physiol Biochem. 2012;30(1):137-50

56. Barnes S. Evolution of the health benefits of soy isoflavones. Proc Soc Exp Biol Med. 1998;217(3):386-92.

57. Si H, Liu D. Genistein, a soy phytoestrogen, upregulates the expression of human endothelial nitric oxide synthase and lowers blood pressure in spontaneously hypertensive rats. J Nutr. 2008;138(2):297-304. 
58. Ferguson JF, Ryan MF, Gibney ER, Brennan L, Roche HM, Reilly MP. Dietary isoflavone intake is associated with evoked responses to inflammatory cardiometabolic stimuli and improved glucose homeostasis in healthy volunteers. Nutr Metab Cardiovasc Dis. 2014;24(9):996-1003.

59. Moreira AP, Texeira TF, Ferreira AB, Peluzio Mdo C, Alfenas Rde C. Influence of a high-fat diet on gut microbiota, intestinal permeability and metabolic endotoxaemia. Br J Nutr. 2012;108(5):801-9.

60. Kim JH, Kim YJ. Effects of genistein in combination with conjugated estrogens on endometrial hyperplasia and metabolic dysfunction in ovariectomized mice. Endocr J. 2015;62(6):531-42.

61. Naaz A, Yellayi S, Zakroczymski MA, Bunick D, Doerge DR, Lubahn DB, Helferich WG, Cooke PS. The soy isoflavone genistein decreases adipose deposition in mice. Endocrinology. 2003;144(8):3315-20.

62. Panneerselvam S, Packirisamy RM, Bobby Z, Elizabeth Jacob S, Sridhar MG. Soy isoflavones (Glycine max) ameliorate hypertriglyceridemia and hepatic steatosis in high fat-fed ovariectomized Wistar rats (an experimental mode of postmenopausal obesity). J Nutr Biochem. 2016;38:57-69.

63. Suzuki T, Hara H. Role of flavonoids in intestinal tight junction regulation. J Nutr Biochem. 2011;22(5):401-8.

64. Moussa L, Bezirard V, Salvador-Cartier C, Bacquie V, Lencina C, Leveque M, Braniste V, Menard S, Theodorou V, Houdeau E. A low dose of fermented soy germ alleviates gut barrier injury, hyperalgesia and faecal protease activity in a rat model of inflammatory bowel disease. PLoS One. 2012;7(11):e49547.

65. Rowland IR, Wiseman H, Sanders TA, Adlercreutz H, Bowey EA. Interindividual variation in metabolism of soy isoflavones and lignans: influence of habitual diet on equol production by the gut microflora. Nutr Cancer. 2000;36(1):27-32.

66. Morselli E, Frank AP, Palmer BF, Rodriguez-Navas C, Criollo A, Clegg DJ. A sexually dimorphic hypothalamic response to chronic high-fat diet consumption. Int J Obes. 2016:40(2):206-9.

67. Liu S, Kilic G, Meyers MS, Navarro G, Wang Y, Oberholzer J, Mauvais-Jarvis F. Oestrogens improve human pancreatic islet transplantation in a mouse model of insulin deficient diabetes. Diabetologia. 2013;56(2):370-81.

68. Chambliss KL, Barrera J, Umetani M, Umetani J, Kim SH, Madak-Erdogan Z, Huang L, Katzenellenbogen BS, Katzenellenbogen JA, Mineo C, et al. Nonnuclear estrogen receptor activation improves hepatic steatosis in female mice. Endocrinology. 2016;157(10):3731-41.

69. Madak-Erdogan Z, Katzenellenbogen BS. Aryl hydrocarbon receptor modulation of estrogen receptor alpha-mediated gene regulation by a multimeric chromatin complex involving the two receptors and the coregulator RIP140. Toxicol Sci. 2012;125(2):401-11.

70. Salpeter SR, Walsh JM, Ormiston TM, Greyber E, Buckley NS, Salpeter EE. Metaanalysis: effect of hormone-replacement therapy on components of the metabolic syndrome in postmenopausal women. Diabetes Obes Metab. 2006;8(5):538-54.

71. Shufelt CL, Merz CN, Prentice RL, Pettinger MB, Rossouw JE, Aroda VR, Kaunitz AM, Lakshminarayan K, Martin LW, Phillips LS, et al. Hormone therapy dose, formulation, route of delivery, and risk of cardiovascular events in women: findings from the Women's Health Initiative observational study. Menopause. 2014;21(3):260-6.

72. Manson JE, Chlebowski RT, Stefanick ML, Aragaki AK, Rossouw JE, Prentice RL, Anderson G, Howard BV, Thomson CA, LaCroix AZ, et al. Menopausal hormone therapy and health outcomes during the intervention and extended poststopping phases of the Women's Health Initiative randomized trials. JAMA. 2013;310(13):1353-68.

73. Burger $\mathrm{H}$. Hormone replacement therapy in the post-Women's Health Initiative era. Report a a meeting held in Funchal, Madeira, February 24-25, 2003. Climacteric. 2003;6(Suppl 1):11-36.

74. Korljan B, Bagatin J, Kokic S, Berovic Matulic N, Barsic Ostojic S, Dekovic A. The impact of hormone replacement therapy on metabolic syndrome components in perimenopausal women. Med Hypotheses. 2010;74(1):162-3.

75. Donley TG, Smith RF, Roy B. Reduced oral contraceptive effectiveness with concurrent antibiotic use: a protocol for prescribing antibiotics to women of childbearing age. Compendium. 1990;11(6):392-6.

76. Baker JM, Al-Nakkash L, Herbst-Kralovetz MM. Estrogen-gut microbiome axis: physiological and clinical implications. Maturitas. 2017;103:45-53.

77. Markle JG, Frank DN, Mortin-Toth S, Robertson CE, Feazel LM, RolleKampczyk U, von Bergen M, McCoy KD, Macpherson AJ, Danska JS. Sex differences in the gut microbiome drive hormone-dependent regulation of autoimmunity. Science. 2013;339(6123):1084-8.

78. Jin G, Asou Y, Ishiyama K, Okawa A, Kanno T, Niwano Y. Proanthocyanidinrich grape seed extract modulates intestinal microbiota in Ovariectomized mice. J Food Sci. 2018;83(4):1149-52.
79. Wang F, Yu P, Gui X, Wang Y, Xue C, Wang J. Sialoglycoprotein isolated from the eggs of Carassius auratus prevents bone loss: an effect associated with the regulation of gut microbiota in ovariectomized rats. Food Funct. 2016:7(12):4764-71.

80. Javurek AB, Spollen WG, Johnson SA, Bivens NJ, Bromert KH, Givan SA, Rosenfeld CS. Effects of exposure to bisphenol a and ethinyl estradiol on the gut microbiota of parents and their offspring in a rodent model. Gut Microbes. 2016;7(6):471-85.

81. Choi S, Hwang YJ, Shin MJ, Yi H. Difference in the gut microbiome between Ovariectomy-induced obesity and diet-induced obesity. J Microbiol Biotechnol. 2017;27(12):2228-36.

82. Chen KLA, Liu X, Zhao YC, Hieronymi K, Rossi G, Auvil LS, Welge M, Bushell C, Smith RL, Carlson KE, et al. Long-term Administration of Conjugated Estrogen and Bazedoxifene Decreased Murine Fecal beta-Glucuronidase activity without impacting overall microbiome community. Sci Rep. 2018;8(1):8166.

83. Yang Y, Qu C, Liang S, Wang G, Han H, Chen N, Wang X, Luo Z, Zhong C, Chen $Y$, et al. Estrogen inhibits the overgrowth of Escherichia coli in the rat intestine under simulated microgravity. Mol Med Rep. 2018;17(2):2313-20.

84. Dakin RS, Walker BR, Seckl JR, Hadoke PW, Drake AJ. Estrogens protect male mice from obesity complications and influence glucocorticoid metabolism. Int J Obes. 2015;39(10):1539-47.

85. Stout MB, Steyn FJ, Jurczak MJ, Camporez JG, Zhu Y, Hawse JR, Jurk D, Palmer AK, Xu M, Pirtskhalava T, et al. 17alpha-estradiol alleviates agerelated metabolic and inflammatory dysfunction in male mice without inducing feminization. J Gerontol A Biol Sci Med Sci. 2017;72(1):3-15.

86. Vieira AT, Castelo PM, Ribeiro DA, Ferreira CM. Influence of Oral and gut microbiota in the health of menopausal women. Front Microbiol. 2017;8:1884.

87. Gordon MN, Osterburg HH, May PC, Finch CE. Effective oral administration of 17 beta-estradiol to female C57BL/6J mice through the drinking water. Biol Reprod. 1986;35(5):1088-95.

88. Reikvam DH, Erofeev A, Sandvik A, Grcic V, Jahnsen FL, Gaustad P, McCoy KD, Macpherson AJ, Meza-Zepeda LA, Johansen FE. Depletion of murine intestinal microbiota: effects on gut mucosa and epithelial gene expression. PLoS One. 2011;6(3):e17996.

89. Magoc T, Salzberg SL. FLASH: fast length adjustment of short reads to improve genome assemblies. Bioinformatics. 2011;27(21):2957-63.

90. Caporaso JG, Kuczynski J, Stombaugh J, Bittinger K, Bushman FD, Costello EK, Fierer N, Pena AG, Goodrich JK, Gordon Jl, et al. QIIME allows analysis of high-throughput community sequencing data. Nat Methods. 2010;7(5):335-6.

91. Edgar RC. Search and clustering orders of magnitude faster than BLAST. Bioinformatics. 2010;26(19):2460-1.

92. Quast C, Pruesse E, Yilmaz P, Gerken J, Schweer T, Yarza P, Peplies J, Glockner FO. The SILVA ribosomal RNA gene database project: improved data processing and web-based tools. Nucleic Acids Res. 2013;41(Database issue):D590-6.

93. Motta V, Trevisi P, Bertolini F, Ribani A, Schiavo G, Fontanesi L, Bosi P. Exploring gastric bacterial community in young pigs. PLoS One. 2017;12(3): e0173029.

94. Kang C, Wang B, Kaliannan K, Wang X, Lang H, Hui S, Huang L, Zhang Y, Zhou M, Chen M, et al. Gut microbiota mediates the protective effects of dietary capsaicin against chronic low-grade inflammation and associated obesity induced by high-fat diet. MBio. 2017;8(3).

95. Chevalier C, Stojanovic O, Colin DJ, Suarez-Zamorano N, Tarallo V, Veyrat-Durebex C, Rigo D, Fabbiano S, Stevanovic A, Hagemann S, et al. Gut microbiota orchestrates energy homeostasis during cold. Cell. 2015;163(6):1360-74.

96. Segata N, Izard J, Waldron L, Gevers D, Miropolsky L, Garrett WS, Huttenhower C. Metagenomic biomarker discovery and explanation. Genome Biol. 2011;12(6):R60.

97. Smilde AK, van der Werf MJ, Bijlsma S, van der Werff-van der Vat BJ, Jellema $\mathrm{RH}$. Fusion of mass spectrometry-based metabolomics data. Anal Chem. 2005;77(20):6729-36.

98. Kelder T, Stroeve JH, Bijlsma S, Radonjic M, Roeselers G. Correlation network analysis reveals relationships between diet-induced changes in human gut microbiota and metabolic health. Nutr Diabetes. 2014;4:e122.

99. Jacobs JP, Goudarzi M, Singh N, Tong M, McHardy $\mathbb{H}$, Ruegger $P$, Asadourian M, Moon BH, Ayson A, Borneman J, et al. A disease-associated microbial and metabolomics state in relatives of pediatric inflammatory bowel disease patients. Cell Mol Gastroenterol Hepatol. 2016;2(6):750-66.

100. Yu K, Mu C, Yang Y, Su Y, Zhu W. Segment-specific responses of intestinal epithelium transcriptome to in-feed antibiotics in pigs. Physiol Genomics. 2017:49(10):582-91. 